\title{
Barnes-type Daehee polynomials
}

\author{
Dae San Kim, Taekyun Kim²*, Takao Komatsu ${ }^{3}$ and Jong-Jin Seo ${ }^{4}$
}

${ }^{*}$ Correspondence: tkkim@kw.ac.kr ${ }^{2}$ Department of Mathematics, Kwangwoon University, Seoul, 139-701, Republic of Korea Full list of author information is available at the end of the article

\section{Abstract}

In this paper, we consider Barnes-type Daehee polynomials of the first kind and of the second kind. From the properties of the Sheffer sequences of these polynomials arising from umbral calculus, we derive new and interesting identities.

MSC: 05A15; 05A40; 11B68; 11B75; 65Q05

\section{Introduction}

In this paper, we consider the polynomials $D_{n}\left(x \mid a_{1}, \ldots, a_{r}\right)$ and $\widehat{D}_{n}\left(x \mid a_{1}, \ldots, a_{r}\right)$ called the Barnes-type Daehee polynomials of the first kind and of the second kind, whose generating functions are given by

$$
\begin{aligned}
& \prod_{j=1}^{r}\left(\frac{\ln (1+t)}{(1+t)^{a_{j}}-1}\right)(1+t)^{x}=\sum_{n=0}^{\infty} D_{n}\left(x \mid a_{1}, \ldots, a_{r}\right) \frac{t^{n}}{n !}, \\
& \prod_{j=1}^{r}\left(\frac{(1+t)^{a_{j}} \ln (1+t)}{(1+t)^{a_{j}}-1}\right)(1+t)^{x}=\sum_{n=0}^{\infty} \widehat{D}_{n}\left(x \mid a_{1}, \ldots, a_{r}\right) \frac{t^{n}}{n !},
\end{aligned}
$$

respectively, where $a_{1}, \ldots, a_{r} \neq 0$. When $x=0, D_{n}\left(a_{1}, \ldots, a_{r}\right)=D_{n}\left(0 \mid a_{1}, \ldots, a_{r}\right)$ and $\widehat{D}_{n}\left(a_{1}\right.$, $\left.\ldots, a_{r}\right)=\widehat{D}_{n}\left(0 \mid a_{1}, \ldots, a_{r}\right)$ are called the Barnes-type Daehee numbers of the first kind and of the second kind, respectively.

Recall that the Daehee polynomials of the first kind and of the second kind of order $r$, denoted by $D_{n}^{(r)}(x)$ and $\widehat{D}_{n}^{(r)}(x)$, respectively, are given by the generating functions to be

$$
\begin{aligned}
& \left(\frac{\ln (1+t)}{t}\right)^{r}(1+t)^{x}=\sum_{n=0}^{\infty} D_{n}^{(r)}(x) \frac{t^{n}}{n !}, \\
& \left(\frac{(1+t) \ln (1+t)}{t}\right)^{r}(1+t)^{x}=\sum_{n=0}^{\infty} \widehat{D}_{n}^{(r)}(x) \frac{t^{n}}{n !}
\end{aligned}
$$

respectively. If $a_{1}=\cdots=a_{r}=1$, then $D_{n}^{(r)}(x)=D_{n}(x \mid \underbrace{1, \ldots, 1}_{r})$ and $\widehat{D}_{n}^{(r)}(x)=\widehat{D}_{n}(x \mid \underbrace{1, \ldots, 1}_{r})$. Daehee polynomials were defined by the second author [1] and have been investigated in [2-4].

In this paper, we consider Barnes-type Daehee polynomials of the first kind and of the second kind. From the properties of the Sheffer sequences of these polynomials arising from umbral calculus, we derive new and interesting identities. 


\section{Umbral calculus}

Let $\mathbb{C}$ be the complex number field and let $\mathcal{F}$ be the set of all formal power series in the variable $t$ :

$$
\mathcal{F}=\left\{f(t)=\sum_{k=0}^{\infty} \frac{a_{k}}{k !} t^{k} \mid a_{k} \in \mathbb{C}\right\} .
$$

Let $\mathbb{P}=\mathbb{C}[x]$ and let $\mathbb{P}^{*}$ be the vector space of all linear functionals on $\mathbb{P} .\langle L \mid p(x)\rangle$ is the action of the linear functional $L$ on the polynomial $p(x)$, and we recall that the vector space operations on $\mathbb{P}^{*}$ are defined by $\langle L+M \mid p(x)\rangle=\langle L \mid p(x)\rangle+\langle M \mid p(x)\rangle,\langle c L \mid p(x)\rangle=c\langle L \mid p(x)\rangle$, where $c$ is a complex constant in $\mathbb{C}$. For $f(t) \in \mathcal{F}$, let us define the linear functional on $\mathbb{P}$ by setting

$$
\left\langle f(t) \mid x^{n}\right\rangle=a_{n} \quad(n \geq 0) .
$$

In particular,

$$
\left\langle t^{k} \mid x^{n}\right\rangle=n ! \delta_{n, k} \quad(n, k \geq 0)
$$

where $\delta_{n, k}$ is the Kronecker symbol.

For $f_{L}(t)=\sum_{k=0}^{\infty} \frac{\left\langle L \mid x^{k}\right\rangle}{k !} t^{k}$, we have $\left\langle f_{L}(t) \mid x^{n}\right\rangle=\left\langle L \mid x^{n}\right\rangle$. That is, $L=f_{L}(t)$. The map $L \mapsto f_{L}(t)$ is a vector space isomorphism from $\mathbb{P}^{*}$ onto $\mathcal{F}$. Henceforth, $\mathcal{F}$ denotes both the algebra of formal power series in $t$ and the vector space of all linear functionals on $\mathbb{P}$, and therefore an element $f(t)$ of $\mathcal{F}$ will be thought of as both a formal power series and a linear functional. We call $\mathcal{F}$ the umbral algebra and the umbral calculus is the study of umbral algebra. The order $O(f(t))$ of a power series $f(t)(\neq 0)$ is the smallest integer $k$ for which the coefficient of $t^{k}$ does not vanish. If $O(f(t))=1$, then $f(t)$ is called a delta series; if $O(f(t))=0$, then $f(t)$ is called an invertible series. For $f(t), g(t) \in \mathcal{F}$ with $O(f(t))=1$ and $O(g(t))=0$, there exists a unique sequence $s_{n}(x)\left(\operatorname{deg} s_{n}(x)=n\right)$ such that $\left\langle g(t) f(t)^{k} \mid s_{n}(x)\right\rangle=n ! \delta_{n, k}$, for $n, k \geq 0$. Such a sequence $s_{n}(x)$ is called the Sheffer sequence for $(g(t), f(t))$, which is denoted by $s_{n}(x) \sim(g(t), f(t))$.

For $f(t), g(t) \in \mathcal{F}$ and $p(x) \in \mathbb{P}$, we have

$$
\langle f(t) g(t) \mid p(x)\rangle=\langle f(t) \mid g(t) p(x)\rangle=\langle g(t) \mid f(t) p(x)\rangle
$$

and

$$
f(t)=\sum_{k=0}^{\infty}\left\langle f(t) \mid x^{k}\right\rangle \frac{t^{k}}{k !}, \quad p(x)=\sum_{k=0}^{\infty}\left\langle t^{k} \mid p(x)\right\rangle \frac{x^{k}}{k !}
$$

[5, Theorem 2.2.5]. Thus, by (7), we get

$$
t^{k} p(x)=p^{(k)}(x)=\frac{d^{k} p(x)}{d x^{k}} \quad \text { and } \quad e^{y t} p(x)=p(x+y) .
$$

Sheffer sequences are characterized by the generating function [5, Theorem 2.3.4]. 
Lemma 1 The sequence $s_{n}(x)$ is Sheffer for $(g(t), f(t))$ if and only if

$$
\frac{1}{g(\bar{f}(t))} e^{y \bar{f}(t)}=\sum_{k=0}^{\infty} \frac{s_{k}(y)}{k !} t^{k} \quad(y \in \mathbb{C})
$$

where $\bar{f}(t)$ is the compositional inverse of $f(t)$.

For $s_{n}(x) \sim(g(t), f(t))$, we have the following equations [5, Theorem 2.3.7, Theorem 2.3.5, Theorem 2.3.9]:

$$
\begin{aligned}
& f(t) s_{n}(x)=n s_{n-1}(x) \quad(n \geq 0), \\
& s_{n}(x)=\sum_{j=0}^{n} \frac{1}{j !}\left\langle g(\bar{f}(t))^{-1} \bar{f}(t)^{j}\left|x^{n}\right| x^{j},\right. \\
& s_{n}(x+y)=\sum_{j=0}^{n}\left(\begin{array}{c}
n \\
j
\end{array}\right) s_{j}(x) p_{n-j}(y),
\end{aligned}
$$

where $p_{n}(x)=g(t) s_{n}(x)$.

Assume that $p_{n}(x) \sim(1, f(t))$ and $q_{n}(x) \sim(1, g(t))$. Then the transfer formula [5, Corollary 3.8.2] is given by

$$
q_{n}(x)=x\left(\frac{f(t)}{g(t)}\right)^{n} x^{-1} p_{n}(x) \quad(n \geq 1) .
$$

For $s_{n}(x) \sim(g(t), f(t))$ and $r_{n}(x) \sim(h(t), l(t))$, assume that

$$
s_{n}(x)=\sum_{m=0}^{n} C_{n, m} r_{m}(x) \quad(n \geq 0) .
$$

Then we have $[5, \mathrm{p} .132]$

$$
C_{n, m}=\frac{1}{m !}\left\langle\frac{h(\bar{f}(t))}{g(\bar{f}(t))} l(\bar{f}(t))^{m} \mid x^{n}\right\rangle .
$$

\section{Main results}

We now note that $D_{n}\left(x \mid a_{1}, \ldots, a_{r}\right)$ is the Sheffer sequence for

$$
g(t)=\prod_{j=1}^{r}\left(\frac{e^{a_{j} t}-1}{t}\right) \text { and } f(t)=e^{t}-1 .
$$

Therefore,

$$
D_{n}\left(x \mid a_{1}, \ldots, a_{r}\right) \sim\left(\prod_{j=1}^{r}\left(\frac{e^{a_{j} t}-1}{t}\right), e^{t}-1\right) .
$$

$\widehat{D}_{n}\left(x \mid a_{1}, \ldots, a_{r}\right)$ is the Sheffer sequence for

$$
g(t)=\prod_{j=1}^{r}\left(\frac{e^{a_{j} t}-1}{t e^{a_{j} t}}\right) \text { and } f(t)=e^{t}-1 .
$$


So,

$$
\widehat{D}_{n}\left(x \mid a_{1}, \ldots, a_{r}\right) \sim\left(\prod_{j=1}^{r}\left(\frac{e^{a_{j} t}-1}{t e^{a_{j} t}}\right), e^{t}-1\right) .
$$

\subsection{Explicit expressions}

Recall that Barnes' multiple Bernoulli polynomials $B_{n}\left(x \mid a_{1}, \ldots, a_{r}\right)$ are defined by the generating function as

$$
\frac{t^{r}}{\prod_{j=1}^{r}\left(e^{a_{j} t}-1\right)} e^{x t}=\sum_{n=0}^{\infty} B_{n}\left(x \mid a_{1}, \ldots, a_{r}\right) \frac{t^{n}}{n !}
$$

where $a_{1}, \ldots, a_{r} \neq 0[6-8]$. Let $(n)_{j}=n(n-1) \cdots(n-j+1)(j \geq 1)$ with $(n)_{0}=1$. The (signed) Stirling numbers of the first kind $S_{1}(n, m)$ are defined by

$$
(x)_{n}=\sum_{m=0}^{n} S_{1}(n, m) x^{m}
$$

\section{Theorem 1}

$$
\begin{aligned}
& D_{n}\left(x \mid a_{1}, \ldots, a_{r}\right)=\sum_{m=0}^{n} S_{1}(n, m) B_{m}\left(x \mid a_{1}, \ldots, a_{r}\right) \\
& =\sum_{j=0}^{n}\left(\sum_{l=j}^{n}\left(\begin{array}{l}
n \\
l
\end{array}\right) S_{1}(l, j) D_{n-l}\left(a_{1}, \ldots, a_{r}\right)\right) x^{j} \\
& =\sum_{m=0}^{n}\left(\begin{array}{c}
n \\
m
\end{array}\right) D_{n-m}\left(a_{1}, \ldots, a_{r}\right)(x)_{m}, \\
& \widehat{D}_{n}\left(x \mid a_{1}, \ldots, a_{r}\right)=\sum_{m=0}^{n} S_{1}(n, m) B_{m}\left(x+a_{1}+\cdots+a_{r} \mid a_{1}, \ldots, a_{r}\right) \\
& =\sum_{j=0}^{n}\left(\sum_{l=j}^{n}\left(\begin{array}{l}
n \\
l
\end{array}\right) S_{1}(l, j) \widehat{D}_{n-l}\left(a_{1}, \ldots, a_{r}\right)\right) x^{j} \\
& =\sum_{m=0}^{n}\left(\begin{array}{c}
n \\
m
\end{array}\right) \widehat{D}_{n-m}\left(a_{1}, \ldots, a_{r}\right)(x)_{m} .
\end{aligned}
$$

Proof Since

$$
\prod_{j=1}^{r}\left(\frac{e^{a_{j} t}-1}{t}\right) D_{n}\left(x \mid a_{1}, \ldots, a_{r}\right) \sim\left(1, e^{t}-1\right)
$$

and

$$
(x)_{n} \sim\left(1, e^{t}-1\right)
$$


we have

$$
\begin{aligned}
D_{n}\left(x \mid a_{1}, \ldots, a_{r}\right) & =\prod_{j=1}^{r}\left(\frac{t}{e^{a_{j} t}-1}\right)(x)_{n} \\
& =\sum_{m=0}^{n} S_{1}(n, m) \prod_{j=1}^{r}\left(\frac{t}{e^{a_{j} t}-1}\right) x^{m} \\
& =\sum_{m=0}^{n} S_{1}(n, m) B_{m}\left(x \mid a_{1}, \ldots, a_{r}\right) .
\end{aligned}
$$

So, we get (16).

Similarly, by

$$
\prod_{j=1}^{r}\left(\frac{e^{a_{j} t}-1}{t e^{a_{j} t}}\right) \widehat{D}_{n}\left(x \mid a_{1}, \ldots, a_{r}\right) \sim\left(1, e^{t}-1\right)
$$

and (23), we have

$$
\begin{aligned}
\widehat{D}_{n}\left(x \mid a_{1}, \ldots, a_{r}\right) & =\prod_{j=1}^{r}\left(\frac{t e^{a_{j} t}}{e^{a_{j} t}-1}\right)(x)_{n} \\
& =\sum_{m=0}^{n} S_{1}(n, m) \prod_{j=1}^{r}\left(\frac{t e^{a_{j} t}}{e^{a_{j} t}-1}\right) x^{m} \\
& =\sum_{m=0}^{n} S_{1}(n, m) e^{\left(a_{1}+\cdots+a_{r}\right) t} \prod_{j=1}^{r}\left(\frac{t}{e^{a_{j} t}-1}\right) x^{m} \\
& =\sum_{m=0}^{n} S_{1}(n, m) B_{m}\left(x+a_{1}+\cdots+a_{r} \mid a_{1}, \ldots, a_{r}\right) .
\end{aligned}
$$

Therefore, we get (19).

By (10) with (13), we get

$$
D_{n}\left(x \mid a_{1}, \ldots, a_{r}\right)=\sum_{j=0}^{n} \frac{1}{j !}\left\langle\prod_{j=1}^{r}\left(\frac{\ln (1+t)}{(1+t)^{a_{j}}-1}\right)(\ln (1+t))^{j} \mid x^{n}\right\rangle x^{j} .
$$

Since

$$
\begin{aligned}
& \left\langle\prod_{j=1}^{r}\left(\frac{\ln (1+t)}{(1+t)^{a_{j}}-1}\right)(\ln (1+t))^{j} \mid x^{n}\right\rangle \\
& \quad=\left\langle\prod_{j=1}^{r}\left(\frac{\ln (1+t)}{(1+t)^{a_{j}}-1}\right) \mid(\ln (1+t))^{j} x^{n}\right\rangle \\
& \quad=\left\langle\prod_{j=1}^{r}\left(\frac{\ln (1+t)}{(1+t)^{a_{j}}-1}\right) \mid j ! \sum_{l=j}^{\infty} S_{1}(l, j) \frac{t^{l}}{l !} x^{n}\right\rangle \\
& \quad=j ! \sum_{l=j}^{n}\left(\begin{array}{c}
n \\
l
\end{array}\right) S_{1}(l, j)\left\langle\prod_{j=1}^{r}\left(\frac{\ln (1+t)}{(1+t)^{a_{j}}-1}\right) \mid x^{n-l}\right\rangle
\end{aligned}
$$




$$
\begin{aligned}
& =j ! \sum_{l=j}^{n}\left(\begin{array}{l}
n \\
l
\end{array}\right) S_{1}(l, j)\left\langle\sum_{i=0}^{\infty} D_{i}\left(a_{1}, \ldots, a_{r}\right) \frac{t^{i}}{i !} \mid x^{n-l}\right\rangle \\
& =j ! \sum_{l=j}^{n}\left(\begin{array}{l}
n \\
l
\end{array}\right) S_{1}(l, j) D_{n-l}\left(a_{1}, \ldots, a_{r}\right),
\end{aligned}
$$

we obtain (17).

Similarly, by (10) with (14), we get

$$
\widehat{D}_{n}\left(x \mid a_{1}, \ldots, a_{r}\right)=\sum_{j=0}^{n} \frac{1}{j !}\left\langle\prod_{j=1}^{r}\left(\frac{(1+t)^{a_{j}} \ln (1+t)}{(1+t)^{a_{j}}-1}\right)(\ln (1+t))^{j} \mid x^{n}\right\rangle x^{j}
$$

Since

$$
\begin{aligned}
& \left\langle\prod_{j=1}^{r}\left(\frac{(1+t)^{a_{j}} \ln (1+t)}{(1+t)^{a_{j}}-1}\right)(\ln (1+t))^{j} \mid x^{n}\right\rangle \\
& \quad=\left\langle\prod_{j=1}^{r}\left(\frac{(1+t)^{a_{j}} \ln (1+t)}{(1+t)^{a_{j}}-1}\right) \mid(\ln (1+t))^{j} x^{n}\right\rangle \\
& \quad=j ! \sum_{l=j}^{n}\left(\begin{array}{l}
n \\
l
\end{array}\right) S_{1}(l, j)\left\langle\prod_{j=1}^{r}\left(\frac{(1+t)^{a_{j}} \ln (1+t)}{(1+t)^{a_{j}}-1}\right) \mid x^{n-l}\right\rangle \\
& \quad=j ! \sum_{l=j}^{n}\left(\begin{array}{l}
n \\
l
\end{array}\right) S_{1}(l, j)\left\langle\sum_{i=0}^{\infty} \widehat{D}_{i}\left(a_{1}, \ldots, a_{r}\right) \frac{t^{i}}{i !} \mid x^{n-l}\right\rangle \\
& \quad=j ! \sum_{l=j}^{n}\left(\begin{array}{l}
n \\
l
\end{array}\right) S_{1}(l, j) \widehat{D}_{n-l}\left(a_{1}, \ldots, a_{r}\right),
\end{aligned}
$$

we obtain (20).

Next, we obtain

$$
\begin{aligned}
D_{n}\left(y \mid a_{1}, \ldots, a_{r}\right) & =\left\langle\sum_{i=0}^{\infty} D_{i}\left(y \mid a_{1}, \ldots, a_{r}\right) \frac{t^{i}}{i !} \mid x^{n}\right\rangle \\
& =\left\langle\prod_{j=1}^{r}\left(\frac{\ln (1+t)}{(1+t)^{a_{j}}-1}\right)(1+t)^{y} \mid x^{n}\right\rangle \\
& =\left\langle\prod_{j=1}^{r}\left(\frac{\ln (1+t)}{(1+t)^{a_{j}}-1}\right) \mid \sum_{m=0}^{\infty}(y)_{m} \frac{t^{m}}{m !} x^{n}\right\rangle \\
& =\sum_{m=0}^{n}(y)_{m}\left(\begin{array}{c}
n \\
m
\end{array}\right)\left\langle\prod_{j=1}^{r}\left(\frac{\ln (1+t)}{(1+t)^{a_{j}}-1}\right) \mid x^{n-m}\right\rangle \\
& =\sum_{m=0}^{n}\left(\begin{array}{c}
n \\
m
\end{array}\right) D_{n-m}\left(a_{1}, \ldots, a_{r}\right)(y)_{m} .
\end{aligned}
$$

Thus, we get the identity (18). 
Similarly,

$$
\begin{aligned}
\widehat{D}_{n}\left(y \mid a_{1}, \ldots, a_{r}\right) & =\left\langle\sum_{i=0}^{\infty} \widehat{D}_{i}\left(y \mid a_{1}, \ldots, a_{r}\right) \frac{t^{i}}{i !} \mid x^{n}\right\rangle \\
& =\left\langle\prod_{j=1}^{r}\left(\frac{(1+t)^{a_{j}} \ln (1+t)}{(1+t)^{a_{j}}-1}\right)(1+t)^{y} \mid x^{n}\right\rangle \\
& =\left\langle\prod_{j=1}^{r}\left(\frac{(1+t)^{a_{j}} \ln (1+t)}{(1+t)^{a_{j}}-1}\right) \mid \sum_{m=0}^{\infty}(y)_{m} \frac{t^{m}}{m !} x^{n}\right\rangle \\
& =\sum_{m=0}^{n}(y)_{m}\left(\begin{array}{c}
n \\
m
\end{array}\right)\left\langle\prod_{j=1}^{r}\left(\frac{(1+t)^{a_{j}} \ln (1+t)}{(1+t)^{a_{j}}-1}\right) \mid x^{n-m}\right\rangle \\
& =\sum_{m=0}^{n}\left(\begin{array}{c}
n \\
m
\end{array}\right) \widehat{D}_{n-m}\left(a_{1}, \ldots, a_{r}\right)(y)_{m} .
\end{aligned}
$$

Thus, we get the identity (21).

\subsection{Sheffer identity}

\section{Theorem 2}

$$
\begin{aligned}
& D_{n}\left(x+y \mid a_{1}, \ldots, a_{r}\right)=\sum_{j=0}^{n}\left(\begin{array}{l}
n \\
j
\end{array}\right) D_{j}\left(x \mid a_{1}, \ldots, a_{r}\right)(y)_{n-j}, \\
& \widehat{D}_{n}\left(x+y \mid a_{1}, \ldots, a_{r}\right)=\sum_{j=0}^{n}\left(\begin{array}{l}
n \\
j
\end{array}\right) \widehat{D}_{j}\left(x \mid a_{1}, \ldots, a_{r}\right)(y)_{n-j} .
\end{aligned}
$$

Proof By (13) with

$$
\begin{aligned}
p_{n}(x) & =\prod_{j=1}^{r}\left(\frac{e^{a_{j} t}-1}{t}\right) D_{n}\left(x \mid a_{1}, \ldots, a_{r}\right) \\
& =(x)_{n} \sim\left(1, e^{t}-1\right),
\end{aligned}
$$

using (11), we have (25).

By (14) with

$$
\begin{aligned}
p_{n}(x) & =\prod_{j=1}^{r}\left(\frac{e^{a_{j} t}-1}{t e^{a_{j} t}}\right) \widehat{D}_{n}\left(x \mid a_{1}, \ldots, a_{r}\right) \\
& =(x)_{n} \sim\left(1, e^{t}-1\right),
\end{aligned}
$$

using (11), we have (26).

\subsection{Difference relations}

\section{Theorem 3}

$$
\begin{aligned}
& D_{n}\left(x+1 \mid a_{1}, \ldots, a_{r}\right)-D_{n}\left(x \mid a_{1}, \ldots, a_{r}\right)=n D_{n-1}\left(x \mid a_{1}, \ldots, a_{r}\right), \\
& \widehat{D}_{n}\left(x+1 \mid a_{1}, \ldots, a_{r}\right)-\widehat{D}_{n}\left(x \mid a_{1}, \ldots, a_{r}\right)=n \widehat{D}_{n-1}\left(x \mid a_{1}, \ldots, a_{r}\right) .
\end{aligned}
$$


Proof By (9) with (13), we get

$$
\left(e^{t}-1\right) D_{n}\left(x \mid a_{1}, \ldots, a_{r}\right)=n D_{n-1}\left(x \mid a_{1}, \ldots, a_{r}\right) .
$$

By (8), we have (27).

Similarly, by (9) with (14), we get

$$
\left(e^{t}-1\right) \widehat{D}_{n}\left(x \mid a_{1}, \ldots, a_{r}\right)=n \widehat{D}_{n-1}\left(x \mid a_{1}, \ldots, a_{r}\right) .
$$

By (8), we have (28).

\subsection{Recurrence}

\section{Theorem 4}

$$
\begin{aligned}
D_{n+1}\left(x \mid a_{1}, \ldots, a_{r}\right)= & x D_{n}\left(x-1 \mid a_{1}, \ldots, a_{r}\right) \\
& -\sum_{m=0}^{n}\left(\sum_{i=m}^{n} \sum_{l=i}^{n} \sum_{j=1}^{r} \frac{1}{i+1}\left(\begin{array}{c}
n \\
l
\end{array}\right)\left(\begin{array}{c}
i+1 \\
m
\end{array}\right) S_{1}(l, i)\right. \\
& \left.\times B_{i+1-m}\left(-a_{j}\right)^{i+1-m} D_{n-l}\left(a_{1}, \ldots, a_{r}\right)\right)(x-1)^{m}, \\
\widehat{D}_{n+1}\left(x \mid a_{1}, \ldots, a_{r}\right)= & \left(x+\sum_{j=1}^{r} a_{j}\right) \widehat{D}_{n}\left(x-1 \mid a_{1}, \ldots, a_{r}\right) \\
& -\sum_{m=0}^{n}\left(\sum_{i=m}^{n} \sum_{l=i}^{n} \sum_{j=1}^{r} \frac{1}{i+1}\left(\begin{array}{c}
n \\
l
\end{array}\right)\left(\begin{array}{c}
i+1 \\
m
\end{array}\right) S_{1}(l, i)\right. \\
& \left.\times B_{i+1-m}\left(-a_{j}\right)^{i+1-m} \widehat{D}_{n-l}\left(a_{1}, \ldots, a_{r}\right)\right)(x-1)^{m},
\end{aligned}
$$

where $B_{n}$ is the nth ordinary Bernoulli number.

Proof By applying

$$
s_{n+1}(x)=\left(x-\frac{g^{\prime}(t)}{g(t)}\right) \frac{1}{f^{\prime}(t)} s_{n}(x)
$$

[5, Corollary 3.7.2] with (13), we get

$$
D_{n+1}\left(x \mid a_{1}, \ldots, a_{r}\right)=x D_{n}\left(x-1 \mid a_{1}, \ldots, a_{r}\right)-e^{-t} \frac{g^{\prime}(t)}{g(t)} D_{n}\left(x \mid a_{1}, \ldots, a_{r}\right) .
$$

Now,

$$
\begin{aligned}
\frac{g^{\prime}(t)}{g(t)} & =(\ln g(t))^{\prime} \\
& =\left(\sum_{j=1}^{r} \ln \left(e^{a_{j} t}-1\right)-r \ln t\right)^{\prime}
\end{aligned}
$$




$$
\begin{aligned}
& =\sum_{j=1}^{r} \frac{a_{j} e^{a_{j} t}}{e^{a_{j} t}-1}-\frac{r}{t} \\
& =\frac{\sum_{j=1}^{r} \prod_{i \neq j}\left(e^{a_{i} t}-1\right)\left(a_{j} t e^{a_{j} t}-e^{a_{j} t}+1\right)}{t \prod_{j=1}^{r}\left(e^{a_{j} t}-1\right)} .
\end{aligned}
$$

Since

$$
\begin{aligned}
\sum_{j=1}^{r} \frac{a_{j} t e^{a_{j} t}}{e^{a_{j} t}-1}-r & =\frac{\sum_{j=1}^{r} \prod_{i \neq j}\left(e^{a_{i} t}-1\right)\left(a_{j} t e^{a_{j} t}-e^{a_{j} t}+1\right)}{\prod_{j=1}^{r}\left(e^{a_{j} t}-1\right)} \\
& =\frac{\frac{1}{2}\left(\sum_{j=1}^{r} a_{1} \cdots a_{j-1} a_{j}^{2} a_{j+1} \cdots a_{r}\right) t^{r+1}+\cdots}{\left(a_{1} \cdots a_{r}\right) t^{r}+\cdots} \\
& =\frac{1}{2}\left(\sum_{j=1}^{r} a_{j}\right) t+\cdots
\end{aligned}
$$

is a series with order $\geq 1$, by (17) we have

$$
\begin{aligned}
& D_{n+1}\left(x \mid a_{1}, \ldots, a_{r}\right) \\
& =x D_{n}\left(x-1 \mid a_{1}, \ldots, a_{r}\right)-e^{-t} \frac{\sum_{j=1}^{r} \frac{a_{j} t e^{a_{j} t}}{e^{a_{j} t}-1}-r}{t} D_{n}\left(x \mid a_{1}, \ldots, a_{r}\right) \\
& =x D_{n}\left(x-1 \mid a_{1}, \ldots, a_{r}\right)-e^{-t} \frac{\sum_{j=1}^{r} \frac{a_{j} t e^{a_{j} t}}{e^{a_{j} t}-1}-r}{t}\left(\sum_{i=0}^{n} \sum_{l=i}^{n}\left(\begin{array}{c}
n \\
l
\end{array}\right) S_{1}(l, i) D_{n-l}\left(a_{1}, \ldots, a_{r}\right) x^{i}\right) \\
& =x D_{n}\left(x-1 \mid a_{1}, \ldots, a_{r}\right)-\sum_{i=0}^{n} \sum_{l=i}^{n}\left(\begin{array}{c}
n \\
l
\end{array}\right) S_{1}(l, i) D_{n-l}\left(a_{1}, \ldots, a_{r}\right) e^{-t}\left(\sum_{j=1}^{r} \frac{a_{j} t e^{a_{j} t}}{e^{a_{j} t}-1}-r\right) \frac{x^{i+1}}{i+1} .
\end{aligned}
$$

Since

$$
\begin{aligned}
e^{-t}\left(\sum_{j=1}^{r} \frac{a_{j} t e^{a_{j} t}}{e^{a_{j} t}-1}-r\right) x^{i+1} & =e^{-t}\left(\sum_{j=1}^{r} \sum_{m=0}^{\infty} \frac{(-1)^{m} B_{m} a_{j}^{m}}{m !} t^{m}-r\right) x^{i+1} \\
& =e^{-t}\left(\sum_{j=1}^{r} \sum_{m=0}^{i+1}\left(\begin{array}{c}
i+1 \\
m
\end{array}\right) B_{m}\left(-a_{j}\right)^{m} x^{i+1-m}-r x^{i+1}\right) \\
& =\sum_{j=1}^{r} \sum_{m=1}^{i+1}\left(\begin{array}{c}
i+1 \\
m
\end{array}\right) B_{m}\left(-a_{j}\right)^{m}(x-1)^{i+1-m} \\
& =\sum_{j=1}^{r} \sum_{m=0}^{i}\left(\begin{array}{c}
i+1 \\
m
\end{array}\right) B_{i+1-m}\left(-a_{j}\right)^{i+1-m}(x-1)^{m},
\end{aligned}
$$

we have

$$
\begin{aligned}
D_{n+1}\left(x \mid a_{1}, \ldots, a_{r}\right)= & x D_{n}\left(x-1 \mid a_{1}, \ldots, a_{r}\right) \\
& -\sum_{i=0}^{n} \sum_{l=i}^{n} \sum_{j=1}^{r} \sum_{m=0}^{i} \frac{1}{i+1}\left(\begin{array}{c}
n \\
l
\end{array}\right)\left(\begin{array}{c}
i+1 \\
m
\end{array}\right) S_{1}(l, i)
\end{aligned}
$$




$$
\begin{aligned}
& \times B_{i+1-m}\left(-a_{j}\right)^{i+1-m} D_{n-l}\left(a_{1}, \ldots, a_{r}\right)(x-1)^{m} \\
= & x D_{n}\left(x-1 \mid a_{1}, \ldots, a_{r}\right) \\
& -\sum_{m=0}^{n}\left(\sum_{i=m}^{n} \sum_{l=i}^{n} \sum_{j=1}^{r} \frac{1}{i+1}\left(\begin{array}{c}
n \\
l
\end{array}\right)\left(\begin{array}{c}
i+1 \\
m
\end{array}\right) S_{1}(l, i)\right. \\
& \left.\times B_{i+1-m}\left(-a_{j}\right)^{i+1-m} D_{n-l}\left(a_{1}, \ldots, a_{r}\right)\right)(x-1)^{m},
\end{aligned}
$$

which is the identity (29).

Next, by applying (31) with (14), we get

$$
\widehat{D}_{n+1}\left(x \mid a_{1}, \ldots, a_{r}\right)=x \widehat{D}_{n}\left(x-1 \mid a_{1}, \ldots, a_{r}\right)-e^{-t} \frac{g^{\prime}(t)}{g(t)} \widehat{D}_{n}\left(x \mid a_{1}, \ldots, a_{r}\right) .
$$

Now,

$$
\begin{aligned}
\frac{g^{\prime}(t)}{g(t)} & =(\ln g(t))^{\prime} \\
& =\left(\sum_{j=1}^{r} \ln \left(e^{a_{j} t}-1\right)-r \ln t-\left(\sum_{j=1}^{r} a_{j}\right) t\right)^{\prime} \\
& =\sum_{j=1}^{r} \frac{a_{j} e^{a_{j} t}}{e^{a_{j} t}-1}-\frac{r}{t}-\sum_{j=1}^{r} a_{j} .
\end{aligned}
$$

By (20) we have

$$
\begin{aligned}
\widehat{D}_{n+1}( & \left.x \mid a_{1}, \ldots, a_{r}\right) \\
= & \left(x+\sum_{j=1}^{r} a_{j}\right) \widehat{D}_{n}\left(x-1 \mid a_{1}, \ldots, a_{r}\right) \\
& -e^{-t} \frac{\sum_{j=1}^{r} \frac{a_{j} t e^{a_{j} t}}{e^{a_{j} t}-1}-r}{t} \widehat{D}_{n}\left(x \mid a_{1}, \ldots, a_{r}\right) \\
= & \left(x+\sum_{j=1}^{r} a_{j}\right) \widehat{D}_{n}\left(x-1 \mid a_{1}, \ldots, a_{r}\right) \\
& -e^{-t} \frac{\sum_{j=1}^{r} \frac{a_{j} t e^{a_{j} t}}{e^{a_{j} t}-1}-r}{t}\left(\sum_{i=0}^{n} \sum_{l=i}^{n}\left(\begin{array}{l}
n \\
l
\end{array}\right) S_{1}(l, i) \widehat{D}_{n-l}\left(a_{1}, \ldots, a_{r}\right) x^{i}\right) \\
= & \left(x+\sum_{j=1}^{r} a_{j}\right) \widehat{D}_{n}\left(x-1 \mid a_{1}, \ldots, a_{r}\right) \\
& -\sum_{i=0}^{n} \sum_{l=i}^{n}\left(\begin{array}{l}
n \\
l
\end{array}\right) S_{1}(l, i) \widehat{D}_{n-l}\left(a_{1}, \ldots, a_{r}\right) e^{-t}\left(\sum_{j=1}^{r} \frac{a_{j} t e^{a_{j} t}}{e^{a_{j} t}-1}-r\right) \frac{x^{i+1}}{i+1} .
\end{aligned}
$$

By (32), we have the identity (30). 


\subsection{Differentiation}

\section{Theorem 5}

$$
\begin{aligned}
& \frac{d}{d x} D_{n}\left(x \mid a_{1}, \ldots, a_{r}\right)=n ! \sum_{l=0}^{n-1} \frac{(-1)^{n-l-1}}{l !(n-l)} D_{l}\left(x \mid a_{1}, \ldots, a_{r}\right), \\
& \frac{d}{d x} \widehat{D}_{n}\left(x \mid a_{1}, \ldots, a_{r}\right)=n ! \sum_{l=0}^{n-1} \frac{(-1)^{n-l-1}}{l !(n-l)} \widehat{D}_{l}\left(x \mid a_{1}, \ldots, a_{r}\right) .
\end{aligned}
$$

Proof We shall use

$$
\frac{d}{d x} s_{n}(x)=\sum_{l=0}^{n-1}\left(\begin{array}{l}
n \\
l
\end{array}\right)\left\langle\bar{f}(t) \mid x^{n-l}\right\rangle s_{l}(x)
$$

(cf. [5, Theorem 2.3.12]). Since

$$
\begin{aligned}
\left\langle\bar{f}(t) \mid x^{n-l}\right\rangle & =\left\langle\ln (1+t) \mid x^{n-l}\right\rangle=\left\langle\sum_{m=1}^{\infty} \frac{(-1)^{m-1} t^{m}}{m} \mid x^{n-l}\right\rangle \\
& =\sum_{m=1}^{n-l} \frac{(-1)^{m-1}}{m}\left\langle t^{m} \mid x^{n-l}\right\rangle \\
& =\sum_{m=1}^{n-l} \frac{(-1)^{m-1}}{m}(n-l) ! \delta_{m, n-l} \\
& =(-1)^{n-l-1}(n-l-1) !
\end{aligned}
$$

with (13), we have

$$
\begin{aligned}
\frac{d}{d x} D_{n}\left(x \mid a_{1}, \ldots, a_{r}\right) & =\sum_{l=0}^{n-1}\left(\begin{array}{l}
n \\
l
\end{array}\right)(-1)^{n-l-1}(n-l-1) ! D_{l}\left(x \mid a_{1}, \ldots, a_{r}\right) \\
& =n ! \sum_{l=0}^{n-1} \frac{(-1)^{n-l-1}}{l !(n-l)} D_{l}\left(x \mid a_{1}, \ldots, a_{r}\right),
\end{aligned}
$$

which is the identity (33). Similarly, with (14), we have the identity (34).

\subsection{More relations}

The classical Cauchy numbers $c_{n}$ are defined by

$$
\frac{t}{\ln (1+t)}=\sum_{n=0}^{\infty} c_{n} \frac{t^{n}}{n !}
$$

(see e.g. $[9,10]$ ).

\section{Theorem 6}

$$
\begin{aligned}
D_{n}\left(x \mid a_{1}, \ldots, a_{r}\right)= & x D_{n-1}\left(x-1 \mid a_{1}, \ldots, a_{r}\right) \\
& +\frac{r}{n} \sum_{l=0}^{n}\left(\begin{array}{l}
n \\
l
\end{array}\right) c_{l} D_{n-l}\left(x-1 \mid a_{1}, \ldots, a_{r}\right)
\end{aligned}
$$




$$
\begin{aligned}
& -\frac{1}{n} \sum_{j=1}^{r} \sum_{l=0}^{n}\left(\begin{array}{l}
n \\
l
\end{array}\right) a_{j} c_{l} D_{n-l}\left(x+a_{j}-1 \mid a_{1}, \ldots, a_{r}, a_{j}\right), \\
\widehat{D}_{n}\left(x \mid a_{1}, \ldots, a_{r}\right)= & \left(x+\sum_{j=1}^{r} a_{j}\right) \widehat{D}_{n-1}\left(x-1 \mid a_{1}, \ldots, a_{r}\right) \\
& +\frac{r}{n} \sum_{l=0}^{n}\left(\begin{array}{l}
n \\
l
\end{array}\right) c_{l} \widehat{D}_{n-l}\left(x-1 \mid a_{1}, \ldots, a_{r}\right) \\
& -\frac{1}{n} \sum_{j=1}^{r} \sum_{l=0}^{n}\left(\begin{array}{l}
n \\
l
\end{array}\right) a_{j} c_{l} \widehat{D}_{n-l}\left(x-1 \mid a_{1}, \ldots, a_{r}, a_{j}\right) .
\end{aligned}
$$

Proof For $n \geq 1$, we have

$$
\begin{aligned}
D_{n}\left(y \mid a_{1}, \ldots, a_{r}\right)= & \sum_{l=0}^{\infty} D_{l}\left(y \mid a_{1}, \ldots, a_{r}\right) \frac{t^{l}}{l !}\left|x^{n}\right\rangle \\
= & \left\langle\prod_{j=1}^{r}\left(\frac{\ln (1+t)}{(1+t)^{a_{j}}-1}\right)(1+t)^{y} \mid x^{n}\right\rangle \\
= & \left\langle\partial_{t}\left(\prod_{j=1}^{r}\left(\frac{\ln (1+t)}{(1+t)^{a_{j}}-1}\right)(1+t)^{y}\right) \mid x^{n-1}\right\rangle \\
= & \left\langle\prod_{j=1}^{r}\left(\frac{\ln (1+t)}{(1+t)^{a_{j}}-1}\right)\left(\partial_{t}(1+t)^{y}\right) \mid x^{n-1}\right\rangle \\
& +\left\langle\left(\partial_{t} \prod_{j=1}^{r}\left(\frac{\ln (1+t)}{(1+t)^{a_{j}}-1}\right)\right)(1+t)^{y} \mid x^{n-1}\right\rangle \\
= & y D_{n-1}\left(y-1 \mid a_{1}, \ldots, a_{r}\right) \\
& +\left\langle\left(\partial_{t} \prod_{j=1}^{r}\left(\frac{\ln (1+t)}{(1+t)^{a_{j}}-1}\right)\right)(1+t)^{y} \mid x^{n-1}\right\rangle .
\end{aligned}
$$

Observe that

$$
\begin{aligned}
\partial_{t} \prod_{j=1}^{r}\left(\frac{\ln (1+t)}{(1+t)^{a_{j}}-1}\right) & =\sum_{j=1}^{r} \prod_{i \neq j}\left(\frac{\ln (1+t)}{(1+t)^{a_{i}}-1}\right) \frac{\frac{1}{1+t}\left((1+t)^{a_{j}}-1\right)-\ln (1+t)\left(a_{j}(1+t)^{a_{j}-1}\right)}{\left((1+t)^{a_{j}}-1\right)^{2}} \\
& =\frac{1}{1+t} \prod_{i=1}^{r}\left(\frac{\ln (1+t)}{(1+t)^{a_{i}}-1}\right) \sum_{j=1}^{r}\left(\frac{1}{\ln (1+t)}-\frac{a_{j}(1+t)^{a_{j}}}{(1+t)^{a_{j}}-1}\right) \\
& =\frac{1}{1+t} \prod_{i=1}^{r}\left(\frac{\ln (1+t)}{(1+t)^{a_{i}}-1}\right) \frac{\sum_{j=1}^{r}\left(\frac{t}{\ln (1+t)}-\frac{a_{j} t(1+t)^{a_{j}}}{(1+t)^{a_{j}}-1}\right)}{t} .
\end{aligned}
$$

Since

$$
\sum_{j=1}^{r}\left(\frac{t}{\ln (1+t)}-\frac{a_{j} t(1+t)^{a_{j}}}{(1+t)^{a_{j}}-1}\right)=-\frac{1}{2}\left(\sum_{j=1}^{r} a_{j}\right) t+\cdots
$$


Kim et al. Advances in Difference Equations 2014, 2014:141

Page 13 of 26

is a series with order $\geq 1$, we have

$$
\begin{aligned}
& \left\langle\left(\partial_{t} \prod_{i=1}^{r}\left(\frac{\ln (1+t)}{(1+t)^{a_{i}}-1}\right)\right)(1+t)^{y} \mid x^{n-1}\right\rangle \\
& =\left\langle\prod_{i=1}^{r}\left(\frac{\ln (1+t)}{(1+t)^{a_{i}}-1}\right)(1+t)^{y-1} \mid \frac{\sum_{j=1}^{r}\left(\frac{t}{\ln (1+t)}-\frac{a_{j} t(1+t)^{a_{j}}}{(1+t)^{a_{j}}-1}\right)}{t} x^{n-1}\right\rangle \\
& =\frac{1}{n} \sum_{j=1}^{r}\left\langle\prod_{i=1}^{r}\left(\frac{\ln (1+t)}{(1+t)^{a_{i}}-1}\right)(1+t)^{y-1} \mid\left(\frac{t}{\ln (1+t)}-\frac{a_{j} t(1+t)^{a_{j}}}{(1+t)^{a_{j}}-1}\right) x^{n}\right\rangle \\
& =\frac{r}{n}\left\langle\prod_{i=1}^{r}\left(\frac{\ln (1+t)}{(1+t)^{a_{i}}-1}\right)(1+t)^{y-1} \mid \frac{t}{\ln (1+t)} x^{n}\right\rangle \\
& -\frac{1}{n} \sum_{j=1}^{r} a_{j}\left\langle\frac{\ln (1+t)}{(1+t)^{a_{j}}-1} \prod_{i=1}^{r}\left(\frac{\ln (1+t)}{(1+t)^{a_{i}}-1}\right)(1+t)^{y+a_{j}-1} \mid \frac{t}{\ln (1+t)} x^{n}\right\rangle \\
& =\frac{r}{n}\left\langle\prod_{i=1}^{r}\left(\frac{\ln (1+t)}{(1+t)^{a_{i}}-1}\right)(1+t)^{y-1} \mid \sum_{l=0}^{\infty} c_{l} \frac{t^{l}}{l !} x^{n}\right\rangle \\
& -\frac{1}{n} \sum_{j=1}^{r} a_{j}\left\langle\frac{\ln (1+t)}{(1+t)^{a_{j}}-1} \prod_{i=1}^{r}\left(\frac{\ln (1+t)}{(1+t)^{a_{i}}-1}\right)(1+t)^{y+a_{j}-1} \mid \sum_{l=0}^{\infty} c_{l} \frac{t^{l}}{l !} x^{n}\right\rangle \\
& =\frac{r}{n} \sum_{l=0}^{n} c_{l}\left(\begin{array}{l}
n \\
l
\end{array}\right)\left\langle\prod_{i=1}^{r}\left(\frac{\ln (1+t)}{(1+t)^{a_{i}}-1}\right)(1+t)^{y-1} \mid x^{n-l}\right\rangle \\
& -\frac{1}{n} \sum_{j=1}^{r} a_{j} \sum_{l=0}^{n} c_{l}\left(\begin{array}{l}
n \\
l
\end{array}\right)\left\langle\frac{\ln (1+t)}{(1+t)^{a_{j}}-1} \prod_{i=1}^{r}\left(\frac{\ln (1+t)}{(1+t)^{a_{i}}-1}\right)(1+t)^{y+a_{j}-1} \mid x^{n-l}\right\rangle \\
& =\frac{r}{n} \sum_{l=0}^{n}\left(\begin{array}{l}
n \\
l
\end{array}\right) c_{l} D_{n-l}\left(y-1 \mid a_{1}, \ldots, a_{r}\right)-\frac{1}{n} \sum_{j=1}^{r} \sum_{l=0}^{n}\left(\begin{array}{l}
n \\
l
\end{array}\right) a_{j} c_{l} D_{n-l}\left(y+a_{j}-1 \mid a_{1}, \ldots, a_{r}, a_{j}\right) .
\end{aligned}
$$

Therefore, we obtain

$$
\begin{aligned}
D_{n}\left(x \mid a_{1}, \ldots, a_{r}\right)= & x D_{n-1}\left(x-1 \mid a_{1}, \ldots, a_{r}\right) \\
& +\frac{r}{n} \sum_{l=0}^{n}\left(\begin{array}{l}
n \\
l
\end{array}\right) c_{l} D_{n-l}\left(x-1 \mid a_{1}, \ldots, a_{r}\right) \\
& -\frac{1}{n} \sum_{j=1}^{r} \sum_{l=0}^{n}\left(\begin{array}{l}
n \\
l
\end{array}\right) a_{j} c_{l} D_{n-l}\left(x+a_{j}-1 \mid a_{1}, \ldots, a_{r}, a_{j}\right),
\end{aligned}
$$

which is the identity (35).

Next, for $n \geq 1$ we have

$$
\begin{aligned}
\widehat{D}_{n}\left(y \mid a_{1}, \ldots, a_{r}\right) & =\left\langle\sum_{l=0}^{\infty} \widehat{D}_{l}\left(y \mid a_{1}, \ldots, a_{r}\right) \frac{t^{l}}{l !} \mid x^{n}\right\rangle \\
& =\left\langle\prod_{j=1}^{r}\left(\frac{(1+t)^{a_{j}} \ln (1+t)}{(1+t)^{a_{j}}-1}\right)(1+t)^{y} \mid x^{n}\right\rangle
\end{aligned}
$$




$$
\begin{aligned}
= & \left\langle\partial_{t}\left(\prod_{j=1}^{r}\left(\frac{(1+t)^{a_{j}} \ln (1+t)}{(1+t)^{a_{j}}-1}\right)(1+t)^{y}\right) \mid x^{n-1}\right\rangle \\
= & \left\langle\prod_{j=1}^{r}\left(\frac{(1+t)^{a_{j}} \ln (1+t)}{(1+t)^{a_{j}}-1}\right)\left(\partial_{t}(1+t)^{y}\right) \mid x^{n-1}\right\rangle \\
& +\left\langle\left(\partial_{t} \prod_{j=1}^{r}\left(\frac{(1+t)^{a_{j}} \ln (1+t)}{(1+t)^{a_{j}}-1}\right)\right)(1+t)^{y} \mid x^{n-1}\right\rangle \\
= & y \widehat{D}_{n-1}\left(y-1 \mid a_{1}, \ldots, a_{r}\right) \\
& +\left\langle\left(\partial_{t} \prod_{j=1}^{r}\left(\frac{(1+t)^{a_{j}} \ln (1+t)}{(1+t)^{a_{j}}-1}\right)\right)(1+t)^{y} \mid x^{n-1}\right\rangle .
\end{aligned}
$$

Observe that

$$
\begin{aligned}
\partial_{t} & \prod_{j=1}^{r}\left(\frac{(1+t)^{a_{j}} \ln (1+t)}{(1+t)^{a_{j}}-1}\right) \\
= & \partial_{t}\left(\prod_{j=1}^{r}\left(\frac{\ln (1+t)}{(1+t)^{a_{j}}-1}\right) \prod_{j=1}^{r}(1+t)^{a_{j}}\right) \\
= & \left(\partial_{t} \prod_{j=1}^{r}\left(\frac{\ln (1+t)}{(1+t)^{a_{j}}-1}\right)\right) \prod_{j=1}^{r}(1+t)^{a_{j}} \\
& +\prod_{j=1}^{r}\left(\frac{\ln (1+t)}{(1+t)^{a_{j}}-1}\right)\left(\partial_{t} \prod_{j=1}^{r}(1+t)^{a_{j}}\right) \\
= & \frac{1}{1+t} \prod_{i=1}^{r}\left(\frac{(1+t)^{a_{i}} \ln (1+t)}{(1+t)^{a_{i}}-1}\right) \frac{\sum_{j=1}^{r}\left(\frac{t}{\ln (1+t)}-\frac{a_{j} t(1+t)^{a_{j}}}{(1+t)^{a_{j}-1}}\right)}{t} \\
& +\frac{1}{1+t} \prod_{i=1}^{r}\left(\frac{(1+t)^{a_{i}} \ln (1+t)}{(1+t)^{a_{i}}-1}\right) \sum_{j=1}^{r} a_{j} .
\end{aligned}
$$

Thus, we have

$$
\begin{aligned}
\left\langle\left(\partial_{t}\right.\right. & \left.\prod_{i=1}^{r}\left(\frac{(1+t)^{a_{i}} \ln (1+t)}{(1+t)^{a_{i}}-1}\right)\right)(1+t)^{y}\left|x^{n-1}\right\rangle \\
= & \left\langle\prod_{i=1}^{r}\left(\frac{(1+t)^{a_{i}} \ln (1+t)}{(1+t)^{a_{i}}-1}\right)(1+t)^{y-1} \mid \frac{\sum_{j=1}^{r}\left(\frac{t}{\ln (1+t)}-\frac{a_{j}(1+t)^{a_{j}}}{(1+t)^{a_{j}-1}}\right)}{t} x^{n-1}\right\rangle \\
& +\left(\sum_{j=1}^{r} a_{j}\right)\left\langle\prod_{i=1}^{r}\left(\frac{(1+t)^{a_{i}} \ln (1+t)}{(1+t)^{a_{i}}-1}\right)(1+t)^{y-1} \mid x^{n-1}\right\rangle \\
= & \left(\sum_{j=1}^{r} a_{j}\right) \widehat{D}_{n-1}\left(y-1 \mid a_{1}, \ldots, a_{r}\right) \\
& +\frac{1}{n}\left\langle\prod_{i=1}^{r}\left(\frac{(1+t)^{a_{i}} \ln (1+t)}{(1+t)^{a_{i}}-1}\right)(1+t)^{y-1} \mid \sum_{j=1}^{r}\left(\frac{t}{\ln (1+t)}-\frac{a_{j} t(1+t)^{a_{j}}}{(1+t)^{a_{j}}-1}\right) x^{n}\right\rangle
\end{aligned}
$$




$$
\begin{aligned}
& =\left(\sum_{j=1}^{r} a_{j}\right) \widehat{D}_{n-1}\left(y-1 \mid a_{1}, \ldots, a_{r}\right)+\frac{r}{n}\left\langle\prod_{i=1}^{r}\left(\frac{(1+t)^{a_{i}} \ln (1+t)}{(1+t)^{a_{i}}-1}\right)(1+t)^{y-1} \mid \frac{t}{\ln (1+t)} x^{n}\right\rangle \\
& -\frac{1}{n} \sum_{j=1}^{r} a_{j}\left\langle\frac{(1+t)^{a_{j}} \ln (1+t)}{(1+t)^{a_{j}}-1} \prod_{i=1}^{r}\left(\frac{(1+t)^{a_{i}} \ln (1+t)}{(1+t)^{a_{i}}-1}\right)(1+t)^{y-1} \mid \frac{t}{\ln (1+t)} x^{n}\right\rangle \\
& =\left(\sum_{j=1}^{r} a_{j}\right) \widehat{D}_{n-1}\left(y-1 \mid a_{1}, \ldots, a_{r}\right)+\frac{r}{n}\left\langle\prod_{i=1}^{r}\left(\frac{(1+t)^{a_{i}} \ln (1+t)}{(1+t)^{a_{i}}-1}\right)(1+t)^{y-1} \mid \sum_{l=0}^{\infty} c_{l} \frac{t^{l}}{l !} x^{n}\right\rangle \\
& -\frac{1}{n} \sum_{j=1}^{r} a_{j}\left\langle\frac{(1+t)^{a_{j}} \ln (1+t)}{(1+t)^{a_{j}}-1} \prod_{i=1}^{r}\left(\frac{(1+t)^{a_{i}} \ln (1+t)}{(1+t)^{a_{i}}-1}\right)(1+t)^{y-1} \mid \sum_{l=0}^{\infty} c_{l} \frac{t^{l}}{l !} x^{n}\right\rangle \\
& =\left(\sum_{j=1}^{r} a_{j}\right) \widehat{D}_{n-1}\left(y-1 \mid a_{1}, \ldots, a_{r}\right) \\
& +\frac{r}{n} \sum_{l=0}^{n} c_{l}\left(\begin{array}{c}
n \\
l
\end{array}\right)\left\langle\prod_{i=1}^{r}\left(\frac{(1+t)^{a_{i}} \ln (1+t)}{(1+t)^{a_{i}}-1}\right)(1+t)^{y-1} \mid x^{n-l}\right\rangle \\
& -\frac{1}{n} \sum_{j=1}^{r} a_{j} \sum_{l=0}^{n} c_{l}\left(\begin{array}{l}
n \\
l
\end{array}\right)\left\langle\frac{(1+t)^{a_{j}} \ln (1+t)}{(1+t)^{a_{j}}-1} \prod_{i=1}^{r}\left(\frac{(1+t)^{a_{i}} \ln (1+t)}{(1+t)^{a_{i}}-1}\right)(1+t)^{y-1} \mid x^{n-l}\right\rangle \\
& =\left(\sum_{j=1}^{r} a_{j}\right) \widehat{D}_{n-1}\left(y-1 \mid a_{1}, \ldots, a_{r}\right)+\frac{r}{n} \sum_{l=0}^{n}\left(\begin{array}{c}
n \\
l
\end{array}\right) c_{l} \widehat{D}_{n-l}\left(y-1 \mid a_{1}, \ldots, a_{r}\right) \\
& -\frac{1}{n} \sum_{j=1}^{r} \sum_{l=0}^{n}\left(\begin{array}{l}
n \\
l
\end{array}\right) a_{j} c_{l} \widehat{D}_{n-l}\left(y-1 \mid a_{1}, \ldots, a_{r}, a_{j}\right) .
\end{aligned}
$$

Therefore, we obtain

$$
\begin{aligned}
\widehat{D}_{n}\left(x \mid a_{1}, \ldots, a_{r}\right)= & \left(x+\sum_{j=1}^{r} a_{j}\right) \widehat{D}_{n-1}\left(x-1 \mid a_{1}, \ldots, a_{r}\right) \\
& +\frac{r}{n} \sum_{l=0}^{n}\left(\begin{array}{c}
n \\
l
\end{array}\right) c_{l} \widehat{D}_{n-l}\left(x-1 \mid a_{1}, \ldots, a_{r}\right) \\
& -\frac{1}{n} \sum_{j=1}^{r} \sum_{l=0}^{n}\left(\begin{array}{c}
n \\
l
\end{array}\right) a_{j} c_{l} \widehat{D}_{n-l}\left(x-1 \mid a_{1}, \ldots, a_{r}, a_{j}\right),
\end{aligned}
$$

which is the identity (36).

\subsection{Relations including the Stirling numbers of the first kind}

Theorem 7 For $n-1 \geq m \geq 1$, we have

$$
\begin{aligned}
\sum_{l=0}^{n-m}\left(\begin{array}{c}
n \\
l
\end{array}\right) S_{1}(n-l, m) D_{l}\left(a_{1}, \ldots, a_{r}\right) & \\
= & \sum_{l=0}^{n-m}\left(\begin{array}{c}
n-1 \\
l
\end{array}\right) S_{1}(n-l-1, m-1) D_{l}\left(-1 \mid a_{1}, \ldots, a_{r}\right) \\
& +\frac{1}{n} \sum_{l=0}^{n-m-1}\left(\begin{array}{c}
n \\
l+1
\end{array}\right) S_{1}(n-l-1, m)
\end{aligned}
$$




$$
\begin{aligned}
& \times\left(r \sum_{i=0}^{l+1}\left(\begin{array}{c}
l+1 \\
i
\end{array}\right) c_{i} D_{l+1-i}\left(-1 \mid a_{1}, \ldots, a_{r}\right)\right. \\
& \left.-\sum_{j=1}^{r} \sum_{i=0}^{l+1}\left(\begin{array}{c}
l+1 \\
i
\end{array}\right) a_{j} c_{i} D_{l+1-i}\left(a_{j}-1 \mid a_{1}, \ldots, a_{r}, a_{j}\right)\right), \\
& \sum_{l=0}^{n-m}\left(\begin{array}{l}
n \\
l
\end{array}\right) S_{1}(n-l, m) \widehat{D}_{l}\left(a_{1}, \ldots, a_{r}\right) \\
& =\sum_{l=0}^{n-m}\left(\begin{array}{c}
n-1 \\
l
\end{array}\right) S_{1}(n-l-1, m-1) \widehat{D}_{l}\left(-1 \mid a_{1}, \ldots, a_{r}\right) \\
& +\frac{1}{n} \sum_{l=0}^{n-m-1}\left(\begin{array}{c}
n \\
l+1
\end{array}\right) S_{1}(n-l-1, m) \\
& \times\left(r \sum_{i=0}^{l+1}\left(\begin{array}{c}
l+1 \\
i
\end{array}\right) c_{i} \widehat{D}_{l+1-i}\left(-1 \mid a_{1}, \ldots, a_{r}\right)\right. \\
& \left.-\sum_{j=1}^{r} \sum_{i=0}^{l+1}\left(\begin{array}{c}
l+1 \\
i
\end{array}\right) a_{j} c_{i} \widehat{D}_{l+1-i}\left(-1 \mid a_{1}, \ldots, a_{r}, a_{j}\right)\right) \\
& +\sum_{l=0}^{n-m-1}\left(\begin{array}{c}
n-1 \\
l
\end{array}\right) S_{1}(n-l-1, m) \sum_{j=1}^{r} a_{j} \widehat{D}_{l}\left(-1 \mid a_{1}, \ldots, a_{r}\right) .
\end{aligned}
$$

Proof We shall compute

$$
\left\langle\prod_{j=1}^{r}\left(\frac{\ln (1+t)}{(1+t)^{a_{j}}-1}\right)(\ln (1+t))^{m} \mid x^{n}\right\rangle
$$

in two different ways. On the one hand,

$$
\begin{aligned}
\left\langle\prod_{j=1}^{r}\left(\frac{\ln (1+t)}{(1+t)^{a_{j}}-1}\right)(\ln (1+t))^{m} \mid x^{n}\right\rangle \\
=\left\langle\prod_{j=1}^{r}\left(\frac{\ln (1+t)}{(1+t)^{a_{j}}-1}\right) \mid(\ln (1+t))^{m} x^{n}\right\rangle \\
=\left\langle\prod_{j=1}^{r}\left(\frac{\ln (1+t)}{(1+t)^{a_{j}}-1}\right) \mid \sum_{l=0}^{\infty} \frac{m !}{(l+m) !} S_{1}(l+m, m) t^{l+m} x^{n}\right\rangle \\
=\sum_{l=0}^{n-m} \frac{m !}{(l+m) !} S_{1}(l+m, m)(n)_{l+m}\left\langle\prod_{j=1}^{r}\left(\frac{\ln (1+t)}{(1+t)^{a_{j}}-1}\right) \mid x^{n-l-m}\right\rangle \\
=\sum_{l=0}^{n-m} m !\left(\begin{array}{c}
n \\
l+m
\end{array}\right) S_{1}(l+m, m) D_{n-l-m}\left(a_{1}, \ldots, a_{r}\right) \\
\quad=\sum_{l=0}^{n-m} m !\left(\begin{array}{l}
n \\
l
\end{array}\right) S_{1}(n-l, m) D_{l}\left(a_{1}, \ldots, a_{r}\right) .
\end{aligned}
$$


On the other hand,

$$
\begin{aligned}
\left\langle\prod_{j=1}^{r}\left(\frac{\ln (1+t)}{(1+t)^{a_{j}}-1}\right)(\ln (1+t))^{m} \mid x^{n}\right\rangle \\
=\left\langle\partial_{t}\left(\prod_{j=1}^{r}\left(\frac{\ln (1+t)}{(1+t)^{a_{j}}-1}\right)(\ln (1+t))^{m}\right) \mid x^{n-1}\right\rangle \\
=\left\langle\left(\partial_{t} \prod_{j=1}^{r}\left(\frac{\ln (1+t)}{(1+t)^{a_{j}}-1}\right)\right)(\ln (1+t))^{m} \mid x^{n-1}\right\rangle \\
\quad+\left\langle\prod_{j=1}^{r}\left(\frac{\ln (1+t)}{(1+t)^{a_{j}}-1}\right) \partial_{t}\left((\ln (1+t))^{m}\right) \mid x^{n-1}\right\rangle .
\end{aligned}
$$

The second term of (39) is equal to

$$
\begin{aligned}
\left\langle\prod_{j=1}^{r}\left(\frac{\ln (1+t)}{(1+t)^{a_{j}}-1}\right) \partial_{t}\left((\ln (1+t))^{m}\right) \mid x^{n-1}\right\rangle & \\
= & m\left\langle\prod_{j=1}^{r}\left(\frac{\ln (1+t)}{(1+t)^{a_{j}}-1}\right)(1+t)^{-1} \mid(\ln (1+t))^{m-1} x^{n-1}\right\rangle \\
= & m\left\langle\prod_{j=1}^{r}\left(\frac{\ln (1+t)}{(1+t)^{a_{j}}-1}\right)(1+t)^{-1} \mid \sum_{l=0}^{n-m} \frac{(m-1) !}{(l+m-1) !} S_{1}(l+m-1, m-1) t^{l+m-1} x^{n-1}\right\rangle \\
= & m \sum_{l=0}^{n-m} \frac{(m-1) !}{(l+m-1) !} S_{1}(l+m-1, m-1)(n-1) l_{l+m-1} \\
& \times\left\langle\prod_{j=1}^{r}\left(\frac{\ln (1+t)}{(1+t)^{a_{j}}-1}\right)(1+t)^{-1} \mid x^{n-l-m}\right\rangle \\
= & m ! \sum_{l=0}^{n-m}\left(\begin{array}{c}
n-1 \\
l+m-1
\end{array}\right) S_{1}(l+m-1, m-1) D_{n-l-m}\left(-1 \mid a_{1}, \ldots, a_{r}\right) \\
= & m ! \sum_{l=0}^{n-m}\left(\begin{array}{c}
n-1 \\
l
\end{array}\right) S_{1}(n-l-1, m-1) D_{l}\left(-1 \mid a_{1}, \ldots, a_{r}\right) .
\end{aligned}
$$

The first term of (39) is equal to

$$
\begin{aligned}
& \left\langle\left(\partial_{t} \prod_{j=1}^{r}\left(\frac{\ln (1+t)}{(1+t)^{a_{j}}-1}\right)\right)(\ln (1+t))^{m} \mid x^{n-1}\right\rangle \\
& \quad=\left\langle\partial_{t} \prod_{j=1}^{r}\left(\frac{\ln (1+t)}{(1+t)^{a_{j}}-1}\right) \mid(\ln (1+t))^{m} x^{n-1}\right\rangle \\
& =\left\langle\partial_{t} \prod_{j=1}^{r}\left(\frac{\ln (1+t)}{(1+t)^{a_{j}}-1}\right) \mid \sum_{l=0}^{n-m-1} \frac{m !}{(l+m) !} S_{1}(l+m, m) t^{l+m} x^{n-1}\right\rangle \\
& =\sum_{l=0}^{n-m-1} \frac{m !}{(l+m) !} S_{1}(l+m, m)(n-1)_{l+m}\left\langle\partial_{t} \prod_{j=1}^{r}\left(\frac{\ln (1+t)}{(1+t)^{a_{j}-1}}\right) \mid x^{n-l-m-1}\right\rangle
\end{aligned}
$$




$$
\begin{aligned}
& =\sum_{l=0}^{n-m-1} m !\left(\begin{array}{c}
n-1 \\
l+m
\end{array}\right) S_{1}(l+m, m) \\
& \times\left\langle\prod_{i=1}^{r}\left(\frac{\ln (1+t)}{(1+t)^{a_{i}}-1}\right)(1+t)^{-1} \mid \frac{\sum_{j=1}^{r}\left(\frac{t}{\ln (1+t)}-\frac{a_{j} t(1+t)^{a_{j}}}{(1+t)^{a_{j}}-1}\right)}{t} x^{n-l-m-1}\right\rangle \\
& =m ! \sum_{l=0}^{n-m-1} \frac{1}{n-l-m}\left(\begin{array}{c}
n-1 \\
l+m
\end{array}\right) S_{1}(l+m, m) \\
& \times\left\langle\prod_{i=1}^{r}\left(\frac{\ln (1+t)}{(1+t)^{a_{i}}-1}\right)(1+t)^{-1} \mid \sum_{j=1}^{r}\left(\frac{t}{\ln (1+t)}-\frac{a_{j} t(1+t)^{a_{j}}}{(1+t)^{a_{j}}-1}\right) x^{n-l-m}\right\rangle \\
& =\frac{m !}{n} \sum_{l=0}^{n-m-1}\left(\begin{array}{c}
n \\
l+1
\end{array}\right) S_{1}(n-1-l, m)\left(r\left\langle\prod_{i=1}^{r}\left(\frac{\ln (1+t)}{(1+t)^{a_{i}}-1}\right)(1+t)^{-1} \mid \frac{t}{\ln (1+t)} x^{l+1}\right\rangle\right. \\
& \left.-\left(\sum_{j=1}^{r} a_{j}\right)\left\langle\frac{\ln (1+t)}{(1+t)^{a_{j}}-1}(1+t)^{a_{j}-1} \prod_{i=1}^{r}\left(\frac{\ln (1+t)}{(1+t)^{a_{i}}-1}\right) \mid \frac{t}{\ln (1+t)} x^{l+1}\right\rangle\right) \\
& =\frac{m !}{n} \sum_{l=0}^{n-m-1}\left(\begin{array}{c}
n \\
l+1
\end{array}\right) S_{1}(n-l-1, m)\left(r\left\langle\prod_{i=1}^{r}\left(\frac{\ln (1+t)}{(1+t)^{a_{i}}-1}\right)(1+t)^{-1} \mid \sum_{i=0}^{l+1} c_{i} \frac{t^{i}}{i !} x^{l+1}\right\rangle\right. \\
& \left.-\left(\sum_{j=1}^{r} a_{j}\right)\left\langle\frac{\ln (1+t)}{(1+t)^{a_{j}}-1}(1+t)^{a_{j}-1} \prod_{i=1}^{r}\left(\frac{\ln (1+t)}{(1+t)^{a_{i}}-1}\right) \mid \sum_{i=0}^{l+1} c_{i} \frac{t^{i}}{i !} x^{l+1}\right\rangle\right) \\
& =\frac{m !}{n} \sum_{l=0}^{n-m-1}\left(\begin{array}{c}
n \\
l+1
\end{array}\right) S_{1}(n-l-1, m)\left(r \sum_{i=0}^{l+1}\left(\begin{array}{c}
l+1 \\
i
\end{array}\right) c_{i} D_{l+1-i}\left(-1 \mid a_{1}, \ldots, a_{r}\right)\right. \\
& \left.-\sum_{j=1}^{r} a_{j} \sum_{i=0}^{l+1}\left(\begin{array}{c}
l+1 \\
i
\end{array}\right) c_{i} D_{l+1-i}\left(a_{j}-1 \mid a_{1}, \ldots, a_{r}, a_{j}\right)\right) .
\end{aligned}
$$

Therefore, we have, for $n-1 \geq m \geq 1$,

$$
\begin{aligned}
m ! & \sum_{l=0}^{n-m}\left(\begin{array}{c}
n \\
l
\end{array}\right) S_{1}(n-l, m) D_{l}\left(a_{1}, \ldots, a_{r}\right) \\
= & m ! \sum_{l=0}^{n-m}\left(\begin{array}{c}
n-1 \\
l
\end{array}\right) S_{1}(n-l-1, m-1) D_{l}\left(-1 \mid a_{1}, \ldots, a_{r}\right) \\
& +\frac{m !}{n} \sum_{l=0}^{n-m-1}\left(\begin{array}{c}
n \\
l+1
\end{array}\right) S_{1}(n-l-1, m) \\
& \times\left(r \sum_{i=0}^{l+1}\left(\begin{array}{c}
l+1 \\
i
\end{array}\right) c_{l+1-i} D_{i}\left(-1 \mid a_{1}, \ldots, a_{r}\right)\right. \\
& \left.-\sum_{j=1}^{r} \sum_{i=0}^{l+1} a_{j}\left(\begin{array}{c}
l+1 \\
i
\end{array}\right) c_{l+1-i} D_{i}\left(a_{j}-1 \mid a_{1}, \ldots, a_{r}, a_{j}\right)\right) .
\end{aligned}
$$

Thus, we get (37).

Next, we shall compute

$$
\left\langle\prod_{j=1}^{r}\left(\frac{(1+t)^{a_{j}} \ln (1+t)}{(1+t)^{a_{j}}-1}\right)(\ln (1+t))^{m} \mid x^{n}\right\rangle
$$


in two different ways. On the one hand,

$$
\begin{aligned}
& \left\langle\prod_{j=1}^{r}\left(\frac{(1+t)^{a_{j}} \ln (1+t)}{(1+t)^{a_{j}}-1}\right)(\ln (1+t))^{m} \mid x^{n}\right\rangle \\
& \quad=\left\langle\prod_{j=1}^{r}\left(\frac{(1+t)^{a_{j}} \ln (1+t)}{(1+t)^{a_{j}}-1}\right) \mid(\ln (1+t))^{m} x^{n}\right\rangle \\
& \quad=\left\langle\prod_{j=1}^{r}\left(\frac{(1+t)^{a_{j}} \ln (1+t)}{(1+t)^{a_{j}}-1}\right) \mid \sum_{l=0}^{\infty} \frac{m !}{(l+m) !} S_{1}(l+m, m) t^{l+m} x^{n}\right\rangle \\
& \quad=\sum_{l=0}^{n-m} \frac{m !}{(l+m) !} S_{1}(l+m, m)(n)_{l+m}\left\langle\prod_{j=1}^{r}\left(\frac{(1+t)^{a_{j}} \ln (1+t)}{(1+t)^{a_{j}}-1}\right) \mid x^{n-l-m}\right\rangle \\
& \quad=\sum_{l=0}^{n-m} m !\left(\begin{array}{c}
n \\
l+m
\end{array}\right) S_{1}(l+m, m) \widehat{D}_{n-l-m}\left(a_{1}, \ldots, a_{r}\right) \\
& =\sum_{l=0}^{n-m} m !\left(\begin{array}{c}
n \\
l
\end{array}\right) S_{1}(n-l, m) \widehat{D}_{l}\left(a_{1}, \ldots, a_{r}\right) .
\end{aligned}
$$

On the other hand,

$$
\begin{aligned}
\left\langle\prod_{j=1}^{r}\left(\frac{(1+t)^{a_{j}} \ln (1+t)}{(1+t)^{a_{j}}-1}\right)(\ln (1+t))^{m} \mid x^{n}\right\rangle & \\
= & \left\langle\partial_{t}\left(\prod_{j=1}^{r}\left(\frac{(1+t)^{a_{j}} \ln (1+t)}{(1+t)^{a_{j}}-1}\right)(\ln (1+t))^{m}\right) \mid x^{n-1}\right\rangle \\
= & \left\langle\left(\partial_{t} \prod_{j=1}^{r}\left(\frac{(1+t)^{a_{j}} \ln (1+t)}{(1+t)^{a_{j}}-1}\right)\right)(\ln (1+t))^{m} \mid x^{n-1}\right\rangle \\
& +\left\langle\prod_{j=1}^{r}\left(\frac{(1+t)^{a_{j}} \ln (1+t)}{(1+t)^{a_{j}}-1}\right) \partial_{t}\left((\ln (1+t))^{m}\right) \mid x^{n-1}\right\rangle .
\end{aligned}
$$

The second term of (40) is equal to

$$
\begin{aligned}
& \left\langle\prod_{j=1}^{r}\left(\frac{(1+t)^{a_{j}} \ln (1+t)}{(1+t)^{a_{j}}-1}\right) \partial_{t}\left((\ln (1+t))^{m}\right) \mid x^{n-1}\right\rangle \\
& =m\left\langle\prod_{j=1}^{r}\left(\frac{(1+t)^{a_{j}} \ln (1+t)}{(1+t)^{a_{j}}-1}\right)(1+t)^{-1} \mid(\ln (1+t))^{m-1} x^{n-1}\right\rangle \\
& =m\left\langle\prod_{j=1}^{r}\left(\frac{(1+t)^{a_{j}} \ln (1+t)}{(1+t)^{a_{j}}-1}\right)(1+t)^{-1} \mid \sum_{l=0}^{n-m} \frac{(m-1) !}{(l+m-1) !} S_{1}(l+m-1, m-1) t^{l+m-1} x^{n-1}\right\rangle \\
& =m \sum_{l=0}^{n-m} \frac{(m-1) !}{(l+m-1) !} S_{1}(l+m-1, m-1)(n-1)_{l+m-1} \\
& \quad \times\left\langle\prod_{j=1}^{r}\left(\frac{(1+t)^{a_{j}} \ln (1+t)}{(1+t)^{a_{j}}-1}\right)(1+t)^{-1} \mid x^{n-l-m}\right\rangle
\end{aligned}
$$




$$
\begin{aligned}
& =m ! \sum_{l=0}^{n-m}\left(\begin{array}{c}
n-1 \\
l+m-1
\end{array}\right) S_{1}(l+m-1, m-1) \widehat{D}_{n-l-m}\left(-1 \mid a_{1}, \ldots, a_{r}\right) \\
& =m ! \sum_{l=0}^{n-m}\left(\begin{array}{c}
n-1 \\
l
\end{array}\right) S_{1}(n-l-1, m-1) \widehat{D}_{l}\left(-1 \mid a_{1}, \ldots, a_{r}\right) .
\end{aligned}
$$

The first term of (40) is equal to

$$
\begin{aligned}
& \left\langle\left(\partial_{t} \prod_{j=1}^{r}\left(\frac{(1+t)^{a_{j}} \ln (1+t)}{(1+t)^{a_{j}}-1}\right)\right)(\ln (1+t))^{m} \mid x^{n-1}\right\rangle \\
& =\left\langle\partial_{t} \prod_{j=1}^{r}\left(\frac{(1+t)^{a_{j}} \ln (1+t)}{(1+t)^{a_{j}}-1}\right) \mid(\ln (1+t))^{m} x^{n-1}\right\rangle \\
& =\left\langle\partial_{t} \prod_{j=1}^{r}\left(\frac{(1+t)^{a_{j}} \ln (1+t)}{(1+t)^{a_{j}}-1}\right) \mid \sum_{l=0}^{n-m-1} \frac{m !}{(l+m) !} S_{1}(l+m, m) t^{l+m} x^{n-1}\right\rangle \\
& =\sum_{l=0}^{n-m-1} \frac{m !}{(l+m) !} S_{1}(l+m, m)(n-1)_{l+m}\left\langle\partial_{t} \prod_{j=1}^{r}\left(\frac{(1+t)^{a_{j}} \ln (1+t)}{(1+t)^{a_{j}}-1}\right) \mid x^{n-l-m-1}\right\rangle .
\end{aligned}
$$

From the proof of (36), we recall

$$
\begin{aligned}
\partial_{t} & \prod_{j=1}^{r}\left(\frac{(1+t)^{a_{j}} \ln (1+t)}{(1+t)^{a_{j}}-1}\right) \\
= & \frac{1}{1+t} \prod_{i=1}^{r}\left(\frac{(1+t)^{a_{i}} \ln (1+t)}{(1+t)^{a_{i}}-1}\right) \frac{\sum_{j=1}^{r}\left(\frac{t}{\ln (1+t)}-\frac{a_{j} t(1+t)^{a_{j}}}{(1+t)^{a_{j}}-1}\right)}{t} \\
& +\frac{1}{1+t} \prod_{i=1}^{r}\left(\frac{(1+t)^{a_{i}} \ln (1+t)}{(1+t)^{a_{i}}-1}\right) \sum_{j=1}^{r} a_{j} .
\end{aligned}
$$

Hence, the first term of (39) is equal to

$$
\begin{aligned}
& \sum_{l=0}^{n-m-1} m !\left(\begin{array}{c}
n-1 \\
l+m
\end{array}\right) S_{1}(l+m, m) \\
& \quad \times\left(\left\langle\prod_{i=1}^{r}\left(\frac{(1+t)^{a_{i}} \ln (1+t)}{(1+t)^{a_{i}}-1}\right)(1+t)^{-1} \mid \frac{\sum_{j=1}^{r}\left(\frac{t}{\ln (1+t)}-\frac{a_{j} t(1+t)^{a_{j}}}{(1+t)^{a_{j}}-1}\right)}{t} x^{n-l-m-1}\right\rangle\right. \\
& \left.+\left(\sum_{j=1}^{r} a_{j}\right)\left\langle\prod_{i=1}^{r}\left(\frac{(1+t)^{a_{i}} \ln (1+t)}{(1+t)^{a_{i}}-1}\right)(1+t)^{-1} \mid x^{n-l-m-1}\right\rangle\right) \\
& =m ! \sum_{l=0}^{n-m-1}\left(\begin{array}{c}
n-1 \\
l
\end{array}\right) S_{1}(n-l-1, m) \\
& \quad \times\left(\left\langle\prod_{i=1}^{r}\left(\frac{(1+t)^{a_{i}} \ln (1+t)}{(1+t)^{a_{i}}-1}\right)(1+t)^{-1} \mid \frac{\sum_{j=1}^{r}\left(\frac{t}{\ln (1+t)}-\frac{a_{j} t(1+t)^{a_{j}}}{(1+t)^{a_{j}}-1}\right)}{t} x^{l}\right\rangle\right.
\end{aligned}
$$


Kim et al. Advances in Difference Equations 2014, 2014:141

Page 21 of 26

http://www.advancesindifferenceequations.com/content/2014/1/141

$$
\begin{aligned}
& \left.+\left(\sum_{j=1}^{r} a_{j}\right)\left\langle\prod_{i=1}^{r}\left(\frac{(1+t)^{a_{i}} \ln (1+t)}{(1+t)^{a_{i}}-1}\right)(1+t)^{-1} \mid x^{l}\right\rangle\right) \\
& =m ! \sum_{l=0}^{n-m-1}\left(\begin{array}{c}
n-1 \\
l
\end{array}\right) S_{1}(n-l-1, m) \\
& \times\left(\frac{1}{l+1}\left\langle\prod_{i=1}^{r}\left(\frac{(1+t)^{a_{i}} \ln (1+t)}{(1+t)^{a_{i}}-1}\right)(1+t)^{-1} \mid \sum_{j=1}^{r}\left(\frac{t}{\ln (1+t)}-\frac{a_{j} t(1+t)^{a_{j}}}{(1+t)^{a_{j}}-1}\right) x^{l+1}\right\rangle\right. \\
& \left.+\left(\sum_{j=1}^{r} a_{j}\right)\left\langle\prod_{i=1}^{r}\left(\frac{(1+t)^{a_{i}} \ln (1+t)}{(1+t)^{a_{i}}-1}\right)(1+t)^{-1} \mid x^{l}\right\rangle\right) \\
& =m ! \sum_{l=0}^{n-m-1}\left(\begin{array}{c}
n-1 \\
l
\end{array}\right) S_{1}(n-l-1, m) \\
& \times\left(\frac{r}{l+1}\left\langle\prod_{i=1}^{r}\left(\frac{(1+t)^{a_{i}} \ln (1+t)}{(1+t)^{a_{i}}-1}\right)(1+t)^{-1} \mid \frac{t}{\ln (1+t)} x^{l+1}\right\rangle\right. \\
& -\frac{1}{l+1}\left(\sum_{j=1}^{r} a_{j}\right)\left\langle\frac{(1+t)^{a_{j}} \ln (1+t)}{(1+t)^{a_{j}}-1} \prod_{i=1}^{r}\left(\frac{(1+t)^{a_{i}} \ln (1+t)}{(1+t)^{a_{i}}-1}\right)(1+t)^{-1} \mid \frac{t}{\ln (1+t)} x^{l+1}\right\rangle \\
& \left.+\left(\sum_{j=1}^{r} a_{j}\right)\left\langle\prod_{i=1}^{r}\left(\frac{(1+t)^{a_{i}} \ln (1+t)}{(1+t)^{a_{i}}-1}\right)(1+t)^{-1} \mid x^{l}\right\rangle\right) \\
& =m ! \sum_{l=0}^{n-m-1}\left(\begin{array}{c}
n-1 \\
l
\end{array}\right) S_{1}(n-l-1, m) \\
& \times\left(\frac{r}{l+1}\left\langle\prod_{i=1}^{r}\left(\frac{(1+t)^{a_{i}} \ln (1+t)}{(1+t)^{a_{i}}-1}\right)(1+t)^{-1} \mid \sum_{i=0}^{l+1} c_{i} \frac{t^{i}}{i !} x^{l+1}\right\rangle\right. \\
& -\frac{1}{l+1}\left(\sum_{j=1}^{r} a_{j}\right)\left\langle\frac{(1+t)^{a_{j}} \ln (1+t)}{(1+t)^{a_{j}}-1} \prod_{i=1}^{r}\left(\frac{(1+t)^{a_{i}} \ln (1+t)}{(1+t)^{a_{i}}-1}\right)(1+t)^{-1} \mid \sum_{i=0}^{l+1} c_{i} \frac{t^{i}}{i !} x^{l+1}\right\rangle \\
& \left.+\left(\sum_{j=1}^{r} a_{j}\right)\left\langle\prod_{i=1}^{r}\left(\frac{(1+t)^{a_{i}} \ln (1+t)}{(1+t)^{a_{i}}-1}\right)(1+t)^{-1} \mid x^{l}\right\rangle\right) \\
& =m ! \sum_{l=0}^{n-m-1}\left(\begin{array}{c}
n-1 \\
l
\end{array}\right) S_{1}(n-l-1, m) \\
& \times\left(\frac{r}{l+1} \sum_{i=0}^{l+1}\left(\begin{array}{c}
l+1 \\
i
\end{array}\right) c_{i} \widehat{D}_{l+1-i}\left(-1 \mid a_{1}, \ldots, a_{r}\right)\right. \\
& \left.-\frac{1}{l+1} \sum_{j=1}^{r} a_{j} \sum_{i=0}^{l+1}\left(\begin{array}{c}
l+1 \\
i
\end{array}\right) c_{i} \widehat{D}_{l+1-i}\left(-1 \mid a_{1}, \ldots, a_{r}, a_{j}\right)+\sum_{j=1}^{r} a_{j} \widehat{D}_{l}\left(-1 \mid a_{1}, \ldots, a_{r}\right)\right) \\
& =\frac{m !}{n} \sum_{l=0}^{n-m-1}\left(\begin{array}{c}
n \\
l+1
\end{array}\right) S_{1}(n-l-1, m) \\
& \times\left(r \sum_{i=0}^{l+1}\left(\begin{array}{c}
l+1 \\
i
\end{array}\right) c_{i} \widehat{D}_{l+1-i}\left(-1 \mid a_{1}, \ldots, a_{r}\right)\right.
\end{aligned}
$$




$$
\begin{aligned}
& \left.-\sum_{j=1}^{r} \sum_{i=0}^{l+1}\left(\begin{array}{c}
l+1 \\
i
\end{array}\right) a_{j} c_{i} \widehat{D}_{l+1-i}\left(-1 \mid a_{1}, \ldots, a_{r}, a_{j}\right)\right) \\
& +m ! \sum_{l=0}^{n-m-1}\left(\begin{array}{c}
n-1 \\
l
\end{array}\right) S_{1}(n-l-1, m) \sum_{j=1}^{r} a_{j} \widehat{D}_{l}\left(-1 \mid a_{1}, \ldots, a_{r}\right)
\end{aligned}
$$

Therefore, we get (38).

\subsection{Relations with the falling factorials}

\section{Theorem 8}

$$
\begin{aligned}
& D_{n}\left(x \mid a_{1}, \ldots, a_{r}\right)=\sum_{m=0}^{n}\left(\begin{array}{l}
n \\
m
\end{array}\right) D_{n-m}\left(a_{1}, \ldots, a_{r}\right)(x)_{m}, \\
& \widehat{D}_{n}\left(x \mid a_{1}, \ldots, a_{r}\right)=\sum_{m=0}^{n}\left(\begin{array}{l}
n \\
m
\end{array}\right) \widehat{D}_{n-m}\left(a_{1}, \ldots, a_{r}\right)(x)_{m} .
\end{aligned}
$$

Proof For (13) and (23), assume that $D_{n}\left(x \mid a_{1}, \ldots, a_{r}\right)=\sum_{m=0}^{n} C_{n, m}(x)_{m}$. By (12), we have

$$
\begin{aligned}
C_{n, m} & =\frac{1}{m !}\left\langle\frac{1}{\prod_{j=1}^{r}\left(\frac{e^{a_{j} \ln (1+t)}-1}{\ln (1+t)}\right)} t^{m} \mid x^{n}\right\rangle \\
& =\frac{1}{m !}\left\langle\prod_{j=1}^{r}\left(\frac{\ln (1+t)}{(1+t)^{a_{j}}-1}\right) \mid t^{m} x^{n}\right\rangle \\
& =\left(\begin{array}{c}
n \\
m
\end{array}\right)\left\langle\prod_{j=1}^{r}\left(\frac{\ln (1+t)}{(1+t)^{a_{j}}-1}\right) \mid x^{n-m}\right\rangle \\
& =\left(\begin{array}{c}
n \\
m
\end{array}\right) D_{n-m}\left(a_{1}, \ldots, a_{r}\right) .
\end{aligned}
$$

Thus, we get the identity (41).

Similarly, for (13) and (23), assume that $\widehat{D}_{n}\left(x \mid a_{1}, \ldots, a_{r}\right)=\sum_{m=0}^{n} C_{n, m}(x)_{m}$. By (12), we have

$$
\begin{aligned}
C_{n, m} & =\frac{1}{m !}\left\langle\frac{1}{\prod_{j=1}^{r}\left(\frac{e^{a_{j} \ln (1+t)}-1}{e^{a_{j} \ln (1+t)} \ln (1+t)}\right)} t^{m} \mid x^{n}\right\rangle \\
& =\frac{1}{m !}\left\langle\prod_{j=1}^{r}\left(\frac{(1+t)^{a_{j}} \ln (1+t)}{(1+t)^{a_{j}}-1}\right) \mid t^{m} x^{n}\right\rangle \\
& =\left(\begin{array}{c}
n \\
m
\end{array}\right)\left\langle\prod_{j=1}^{r}\left(\frac{(1+t)^{a_{j}} \ln (1+t)}{(1+t)^{a_{j}}-1}\right) \mid x^{n-m}\right\rangle \\
& =\left(\begin{array}{c}
n \\
m
\end{array}\right) \widehat{D}_{n-m}\left(a_{1}, \ldots, a_{r}\right) .
\end{aligned}
$$

Thus, we get the identity (42). 


\subsection{Relations with higher-order Frobenius-Euler polynomials}

For $\lambda \in \mathbb{C}$ with $\lambda \neq 1$, the Frobenius-Euler polynomials of order $r, H_{n}^{(r)}(x \mid \lambda)$ are defined by the generating function

$$
\left(\frac{1-\lambda}{e^{t}-\lambda}\right)^{r} e^{x t}=\sum_{n=0}^{\infty} H_{n}^{(r)}(x \mid \lambda) \frac{t^{n}}{n !}
$$

(see e.g. $[11,12])$.

\section{Theorem 9}

$$
\begin{aligned}
D_{n}\left(x \mid a_{1}, \ldots, a_{r}\right)= & \sum_{m=0}^{n}\left(\sum_{j=0}^{n-m} \sum_{l=0}^{n-m-j}\left(\begin{array}{c}
s \\
j
\end{array}\right)\left(\begin{array}{c}
n-j \\
l
\end{array}\right)(n)_{j}\right. \\
& \left.\times(1-\lambda)^{-j} S_{1}(n-j-l, m) D_{l}\left(a_{1}, \ldots, a_{r}\right)\right) H_{m}^{(s)}(x \mid \lambda), \\
\widehat{D}_{n}\left(x \mid a_{1}, \ldots, a_{r}\right)= & \sum_{m=0}^{n}\left(\sum_{j=0}^{n-m} \sum_{l=0}^{n-m-j}\left(\begin{array}{c}
s \\
j
\end{array}\right)\left(\begin{array}{c}
n-j \\
l
\end{array}\right)(n)_{j}\right. \\
& \left.\times(1-\lambda)^{-j} S_{1}(n-j-l, m) \widehat{D}_{l}\left(a_{1}, \ldots, a_{r}\right)\right) H_{m}^{(s)}(x \mid \lambda) .
\end{aligned}
$$

Proof For (13) and

$$
H_{n}^{(s)}(x \mid \lambda) \sim\left(\left(\frac{e^{t}-\lambda}{1-\lambda}\right)^{s}, t\right),
$$

assume that $D_{n}\left(x \mid a_{1}, \ldots, a_{r}\right)=\sum_{m=0}^{n} C_{n, m} H_{m}^{(s)}(x \mid \lambda)$. By (12), similarly to the proof of (37), we have

$$
\begin{aligned}
C_{n, m} & =\frac{1}{m !}\left\langle\frac{\left(\frac{e^{\ln (1+t)}-\lambda}{1-\lambda}\right)^{s}}{\prod_{j=1}^{r}\left(\frac{e_{j} a_{j} \ln (1+t)}{\ln (1+t)}\right)}(\ln (1+t))^{m} \mid x^{n}\right\rangle \\
& =\frac{1}{m !(1-\lambda)^{s}}\left\langle\prod_{j=1}^{r}\left(\frac{\ln (1+t)}{(1+t)^{a_{j}}-1}\right)(\ln (1+t))^{m}(1-\lambda+t)^{s} \mid x^{n}\right\rangle \\
& =\frac{1}{m !(1-\lambda)^{s}}\left\langle\prod_{j=1}^{r}\left(\frac{\ln (1+t)}{(1+t)^{a_{j}}-1}\right)(\ln (1+t))^{m} \mid \sum_{i=0}^{\min \{s, n\}}\left(\begin{array}{l}
s \\
i
\end{array}\right)(1-\lambda)^{s-i} t^{i} x^{n}\right\rangle \\
& =\frac{1}{m !(1-\lambda)^{s}} \sum_{i=0}^{n-m}\left(\begin{array}{c}
s \\
i
\end{array}\right)(1-\lambda)^{s-i}(n)_{i}\left\langle\prod_{j=1}^{r}\left(\frac{\ln (1+t)}{(1+t)^{a_{j}}-1}\right) \mid(\ln (1+t))^{m} x^{n-i}\right\rangle \\
& =\frac{1}{m !(1-\lambda)^{s}} \sum_{i=0}^{n-m}\left(\begin{array}{c}
s \\
i
\end{array}\right)(1-\lambda)^{s-i}(n)_{i} \sum_{l=0}^{n-m-i} m !\left(\begin{array}{c}
n-i \\
l
\end{array}\right) S_{1}(n-i-l, m) D_{l}\left(a_{1}, \ldots, a_{r}\right) \\
& =\sum_{i=0}^{n-m} \sum_{l=0}^{n-m-i}\left(\begin{array}{c}
s \\
i
\end{array}\right)\left(\begin{array}{c}
n-i \\
l
\end{array}\right)(n)_{i}(1-\lambda)^{-i} S_{1}(n-i-l, m) D_{l}\left(a_{1}, \ldots, a_{r}\right) .
\end{aligned}
$$

Thus, we get the identity (43). 
Next, for (14) and (45), assume that $\widehat{D}_{n}\left(x \mid a_{1}, \ldots, a_{r}\right)=\sum_{m=0}^{n} C_{n, m} H_{m}^{(s)}(x \mid \lambda)$. By (12), similarly to the proof of (38), we have

$$
\begin{aligned}
C_{n, m} & =\frac{1}{m !}\left\langle\frac{\left(\frac{e^{\ln (1+t)}-\lambda}{1-\lambda}\right)^{s}}{\prod_{j=1}^{r}\left(\frac{e^{a_{j} \ln (1+t)}-1}{e^{a_{j} \ln (1+t)} \ln (1+t)}\right)}(\ln (1+t))^{m} \mid x^{n}\right\rangle \\
& =\frac{1}{m !(1-\lambda)^{s}}\left\langle\prod_{j=1}^{r}\left(\frac{(1+t)^{a_{j}} \ln (1+t)}{(1+t)^{a_{j}}-1}\right)(\ln (1+t))^{m} \mid(1-\lambda+t)^{s} x^{n}\right\rangle \\
& =\frac{1}{m !(1-\lambda)^{s}}\left\langle\prod_{j=1}^{r}\left(\frac{(1+t)^{a_{j}} \ln (1+t)}{(1+t)^{a_{j}}-1}\right)(\ln (1+t))^{m} \mid \sum_{i=0}^{\min \{s, n\}}\left(\begin{array}{l}
s \\
i
\end{array}\right)(1-\lambda)^{s-i} t^{i} x^{n}\right\rangle \\
& =\frac{1}{m !(1-\lambda)^{s}} \sum_{i=0}^{n-m}\left(\begin{array}{c}
s \\
i
\end{array}\right)(1-\lambda)^{s-i}(n)_{i}\left\langle\prod_{j=1}^{r}\left(\frac{(1+t)^{a_{j}} \ln (1+t)}{(1+t)^{a_{j}}-1}\right) \mid(\ln (1+t))^{m} x^{n-i}\right\rangle \\
& =\frac{1}{m !(1-\lambda)^{s}} \sum_{i=0}^{n-m}\left(\begin{array}{c}
s \\
i
\end{array}\right)(1-\lambda)^{s-i}(n)_{i} \sum_{l=0}^{n-m-i} m !\left(\begin{array}{c}
n-i \\
l
\end{array}\right) S_{1}(n-i-l, m) \widehat{D}_{l}\left(a_{1}, \ldots, a_{r}\right) \\
& =\sum_{i=0}^{n-m} \sum_{l=0}^{n-m-i}\left(\begin{array}{l}
s \\
i
\end{array}\right)\left(\begin{array}{c}
n-i \\
l
\end{array}\right)(n)_{i}(1-\lambda)^{-i} S_{1}(n-i-l, m) \widehat{D}_{l}\left(a_{1}, \ldots, a_{r}\right) .
\end{aligned}
$$

Thus, we get the identity (44).

\subsection{Relations with higher-order Bernoulli polynomials}

Bernoulli polynomials $\mathfrak{B}_{n}^{(r)}(x)$ of order $r$ are defined by

$$
\left(\frac{t}{e^{t}-1}\right)^{r} e^{x t}=\sum_{n=0}^{\infty} \frac{\mathfrak{B}_{n}^{(r)}(x)}{n !} t^{n}
$$

(see e.g. [5, Section 2.2]). In addition, Cauchy numbers of the first kind $\mathfrak{C}_{n}^{(r)}$ of order $r$ are defined by

$$
\left(\frac{t}{\ln (1+t)}\right)^{r}=\sum_{n=0}^{\infty} \frac{\mathfrak{C}_{n}^{(r)}}{n !} t^{n}
$$

(see e.g. $[13,(2.1)],[14,(6)])$.

\section{Theorem 10}

$$
\begin{aligned}
& D_{n}\left(x \mid a_{1}, \ldots, a_{r}\right) \\
& \quad=\sum_{m=0}^{n}\left(\sum_{i=0}^{n-m} \sum_{l=0}^{n-m-i}\left(\begin{array}{c}
n \\
i
\end{array}\right)\left(\begin{array}{c}
n-i \\
l
\end{array}\right) \mathfrak{C}_{i}^{(s)} S_{1}(n-i-l, m) D_{l}\left(a_{1}, \ldots, a_{r}\right)\right) \mathfrak{B}_{m}^{(s)}(x), \\
& \widehat{D}_{n}\left(x \mid a_{1}, \ldots, a_{r}\right) \\
& \quad=\sum_{m=0}^{n}\left(\sum_{i=0}^{n-m} \sum_{l=0}^{n-m-i}\left(\begin{array}{c}
n \\
i
\end{array}\right)\left(\begin{array}{c}
n-i \\
l
\end{array}\right) \mathfrak{C}_{i}^{(s)} S_{1}(n-i-l, m) \widehat{D}_{l}\left(a_{1}, \ldots, a_{r}\right)\right) \mathfrak{B}_{m}^{(s)}(x) .
\end{aligned}
$$


Proof For (13) and

$$
\mathfrak{B}_{n}^{(s)}(x) \sim\left(\left(\frac{e^{t}-1}{t}\right)^{s}, t\right),
$$

assume that $D_{n}\left(x \mid a_{1}, \ldots, a_{r}\right)=\sum_{m=0}^{n} C_{n, m} \mathfrak{B}_{m}^{(s)}(x)$. By (12), similarly to the proof of (37), we have

$$
\begin{aligned}
C_{n, m} & =\frac{1}{m !}\left\langle\frac{\left(\frac{e^{\ln (1+t)}-1}{\ln (1+t)}\right)^{s}}{\prod_{j=1}^{r}\left(\frac{e^{a_{j} \ln (1+t)}-1}{\ln (1+t)}\right)}(\ln (1+t))^{m} \mid x^{n}\right\rangle \\
& =\frac{1}{m !}\left\langle\prod_{j=1}^{r}\left(\frac{\ln (1+t)}{(1+t)^{a_{j}}-1}\right)(\ln (1+t))^{m} \mid\left(\frac{t}{\ln (1+t)}\right)^{s} x^{n}\right\rangle \\
& =\frac{1}{m !}\left\langle\prod_{j=1}^{r}\left(\frac{\ln (1+t)}{(1+t)^{a_{j}}-1}\right)(\ln (1+t))^{m} \mid \sum_{i=0}^{\infty} \mathfrak{C}_{i}^{(s)} \frac{t^{i}}{i !} x^{n}\right\rangle \\
& =\frac{1}{m !} \sum_{i=0}^{n-m} \mathfrak{C}_{i}^{(s)}\left(\begin{array}{c}
n \\
i
\end{array}\right)\left\langle\prod_{j=1}^{r}\left(\frac{\ln (1+t)}{(1+t)^{a_{j}}-1}\right)(\ln (1+t))^{m} \mid x^{n-i}\right\rangle \\
& =\frac{1}{m !} \sum_{i=0}^{n-m} \mathfrak{C}_{i}^{(s)}\left(\begin{array}{c}
n \\
i
\end{array}\right) \sum_{l=0}^{n-m-i} m !\left(\begin{array}{c}
n-i \\
l
\end{array}\right) S_{1}(n-i-l, m) D_{l}\left(a_{1}, \ldots, a_{r}\right) \\
& =\sum_{i=0}^{n-m} \sum_{l=0}^{n-m-i}\left(\begin{array}{c}
n \\
i
\end{array}\right)\left(\begin{array}{c}
n-i \\
l
\end{array}\right) \mathfrak{C}_{i}^{(s)} S_{1}(n-i-l, m) D_{l}\left(a_{1}, \ldots, a_{r}\right) .
\end{aligned}
$$

Thus, we get the identity (46).

Next, for (13) and (48), assume that $\widehat{D}_{n}\left(x \mid a_{1}, \ldots, a_{r}\right)=\sum_{m=0}^{n} C_{n, m} \mathfrak{B}_{m}^{(s)}(x)$. By (12), similarly to the proof of (38), we have

$$
\begin{aligned}
C_{n, m} & =\frac{1}{m !}\left\langle\frac{\left(\frac{e^{\ln (1+t)}-1}{\ln (1+t)}\right)^{s}}{\prod_{j=1}^{r}\left(\frac{e^{a_{j} \ln (1+t)}-1}{e^{a_{j} \ln (1+t)} \ln (1+t)}\right)}(\ln (1+t))^{m} \mid x^{n}\right\rangle \\
& =\frac{1}{m !}\left\langle\prod_{j=1}^{r}\left(\frac{(1+t)^{a_{j}} \ln (1+t)}{(1+t)^{a_{j}}-1}\right)(\ln (1+t))^{m} \mid\left(\frac{t}{\ln (1+t)}\right)^{s} x^{n}\right\rangle \\
& =\frac{1}{m !}\left\langle\prod_{j=1}^{r}\left(\frac{(1+t)^{a_{j}} \ln (1+t)}{(1+t)^{a_{j}}-1}\right)(\ln (1+t))^{m} \mid \sum_{i=0}^{\infty} \mathfrak{C}_{i}^{(s)} \frac{t^{i}}{i !} x^{n}\right\rangle \\
& =\frac{1}{m !} \sum_{i=0}^{n-m} \mathfrak{C}_{i}^{(s)}\left(\begin{array}{c}
n \\
i
\end{array}\right)\left\langle\prod_{j=1}^{r}\left(\frac{(1+t)^{a_{j}} \ln (1+t)}{(1+t)^{a_{j}}-1}\right)(\ln (1+t))^{m} \mid x^{n-i}\right\rangle \\
& =\frac{1}{m !} \sum_{i=0}^{n-m} \mathfrak{C}_{i}^{(s)}\left(\begin{array}{c}
n \\
i
\end{array}\right) \sum_{l=0}^{n-m-i} m !\left(\begin{array}{c}
n-i \\
l
\end{array}\right) S_{1}(n-i-l, m) \widehat{D}_{l}\left(a_{1}, \ldots, a_{r}\right) \\
& =\sum_{i=0}^{n-m} \sum_{l=0}^{n-m-i}\left(\begin{array}{c}
n \\
i
\end{array}\right)\left(\begin{array}{c}
n-i \\
l
\end{array}\right) \mathfrak{C}_{i}^{(s)} S_{1}(n-i-l, m) \widehat{D}_{l}\left(a_{1}, \ldots, a_{r}\right) .
\end{aligned}
$$

Thus, we get the identity (47). 


\section{Competing interests}

The authors declare that they have no competing interests.

\section{Authors' contributions}

All authors contributed equally to this work. All authors read and approved the final manuscript.

\section{Author details}

${ }^{1}$ Department of Mathematics, Sogang University, Seoul, 121-742, Republic of Korea. ${ }^{2}$ Department of Mathematics, Kwangwoon University, Seoul, 139-701, Republic of Korea. ${ }^{3}$ Graduate School of Science and Technology, Hirosaki University, Hirosaki, 036-8561, Japan. ${ }^{4}$ Department of Applied Mathematics, Pukyong National University, Pusan, 608-739, Republic of Korea.

\section{Acknowledgements}

This work was supported by the National Research Foundation of Korea (NRF) grant funded by the Korean government (MOE) (No. 2012R1A1A2003786). The authors would like to thank the referee for his valuable comments.

Received: 14 January 2014 Accepted: 30 April 2014 Published: 09 May 2014

\section{References}

1. Kim, T: An invariant $p$-adic integral associated with Daehee numbers. Integral Transforms Spec. Funct. 13, 65-69 $(2002)$

2. Kim, DS, Kim, T, Rim, S-H: On the associated sequence of special polynomials. Adv. Stud. Contemp. Math. (Kyungshang) 23, 355-366 (2013)

3. Ozden, H, Cangul, IN, Simsek, Y: Remarks on q-Bernoulli numbers associated with Daehee numbers. Adv. Stud. Contemp. Math. (Kyungshang) 18, 41-48 (2009)

4. Park, J-W, Rim, S-H, Kwon, J: The twisted Daehee numbers and polynomials. Adv. Differ. Equ. 2014, 1 (2014)

5. Roman, S: The Umbral Calculus. Dover, New York (2005)

6. Kim, T: On Euler-Barnes multiple zeta functions. Russ. J. Math. Phys. 10, 261-267 (2003)

7. Kim, T: Barnes-type multiple q-zeta functions and q-Euler polynomials. J. Phys. A 43, 255201 (2010)

8. Bayad, A, Kim, T, Kim, WJ, Lee, SH: Arithmetic properties of q-Barnes polynomials. J. Comput. Anal. Appl. 15, 111-117 (2013)

9. Comtet, L: Advanced Combinatorics. Reidel, Dordrecht (1974)

10. Komatsu, T: Poly-Cauchy numbers. Kyushu J. Math. 67, 143-153 (2013)

11. Kim, DS, Kim, T: Some identities of Frobenius-Euler polynomials arising from umbral calculus. Adv. Differ. Equ. 2012, $196(2012)$

12. Kim, DS, Kim, T, Lee, S-H: Poly-Bernoulli polynomials arising from umbral calculus. arXiv:1306.6697

13. Carlitz, L: A note on Bernoulli and Euler polynomials of the second kind. Scr. Math. 25, 323-330 (1961)

14. Liang, $\mathrm{H}$, Wuyungaowa: Identities involving generalized harmonic numbers and other special combinatorial sequences. J. Integer Seq. 15, Article 12.9.6 (2012)

10.1186/1687-1847-2014-141

Cite this article as: Kim et al.: Barnes-type Daehee polynomials. Advances in Difference Equations 2014, $2014: 141$

\section{Submit your manuscript to a SpringerOpen ${ }^{\circ}$ journal and benefit from:}

- Convenient online submission

- Rigorous peer review

- Immediate publication on acceptance

- Open access: articles freely available online

- High visibility within the field

- Retaining the copyright to your article 